\title{
PD-L1 inhibits acute and chronic pain by suppressing nociceptive neuron activity via PD-1
}

\author{
Gang Chen ${ }^{1,2,6}$, Yong Ho Kim ${ }^{1,3,6}$, Hui Li ${ }^{4}$, Hao Luo ${ }^{1,4}$, Da-Lu Liu ${ }^{1}$, Zhi-Jun Zhang ${ }^{1}$, Mark \\ Lay $^{1}$, Wonseok Chang ${ }^{1}$, Yu-Qiu Zhang ${ }^{4}$, and Ru-Rong $\mathrm{Ji}^{1,4,5}$ \\ ${ }^{1}$ Department of Anesthesiology, Duke University Medical Center, Durham, North Carolina, 27710 \\ ${ }^{2}$ Key Laboratory of Neuroregeneration of Jiangsu and Ministry of Education, Co-Innovation \\ Center of Neuroregeneration, Nantong University, Nantong, Jiangsu 226001, China \\ ${ }^{3}$ Department of Physiology, College of Medicine, Gachon University, Incheon, Korea \\ ${ }^{4}$ Institute of Neurobiology, Institutes of Brain Science and State Key Laboratory of Medical \\ Neurobiology, Collaborative Innovation Center for Brain Science, Fudan University, Shanghai \\ 200032, China
}

${ }^{5}$ Department of Neurobiology, Duke University Medical Center, Durham, North Carolina, 27710

\section{Abstract}

\begin{abstract}
Programmed cell death ligand-1 (PD-L1) is typically produced by cancer cells and suppresses immunity through PD-1 receptor expressed on T cells. However, the role of PD-L1/PD-1 in regulating pain and neuronal function is unclear. Here we report that both melanoma and normal neural tissues including dorsal root ganglia (DRG) produce PD-L1 that can potently inhibit acute and chronic pain. Intraplantar injection of PD-L1 evokes analgesia in naïve mice via PD-1, whereas PD-L1 neutralization or PD-1 blockade induces mechanical allodynia. Mice lacking Pd1 exhibit thermal and mechanical hypersensitivity. PD-1 activation in DRG nociceptive neurons by PD-L1 induces SHP-1 phosphorylation, inhibits sodium channels, and causes hyperpolarization through activation of TREK2 $\mathrm{K}^{+}$channels. PD-L1 also potently suppresses nociceptive neuron excitability of human DRGs. Remarkably, blocking PD-L1 or PD-1 elicits spontaneous pain and allodynia in melanoma-bearing mice. Our findings identify a previously unrecognized role of PDL1 as an endogenous pain inhibitor and a neuromodulator.
\end{abstract}

\footnotetext{
"Correspondence should be addressed: Ru-Rong Ji, PhD, Department of Anesthesiology, Duke University Medical Center, Durham, North Carolina, 27710 Tel: 919-684-9387, ru-rong.ji@duke.edu, Or Yu-Qiu Zhang, Institute of Neurobiology, Institutes of Brain Science and State Key Laboratory of Medical Neurobiology, Collaborative Innovation Center for Brain Science, Fudan University, Shanghai 200032, China, yuqiuzhang@ fudan.edu.cn.

${ }^{6}$ These authors contribute equally to this study.

All the authors have no financial interests in this study.

COMPETING FINANICIAL INTERESTS

The authors declare no competing financial interests.

Note: Supplementary Information is also available in the online version of the paper.

AUTHOR CONTRIBUTIONS

G.C. developed the project, performed behavioral and histochemical experiments, and prepared the final figures. Y.H.K. conducted electrophysiology in mouse and human DRG neurons. Hui.L. and Hao.L. performed spinal cord recordings and behavioral test in bonce caner model under the guidance of Y.-Q.Z. D.L. performed recordings in whole-mount mouse DRGs. Z.J.Z contributed to histochemistry in Pd1 knockout mice. M.L. did some in situ hybridization experiment. W.C conducted some electrophysiology in mouse DRG neurons. R.-R. J. and Y.-Q.Z. supervised the project. R.-R. J., G.C. and Y.-Q.Z. wrote the paper.
} 


\section{INTRODUCTION}

Cancer pain dramatically impairs the quality of life in patients. Breast, lung, and prostate cancers frequently metastasize to multiple bones and cause bone cancer pain, by releasing algogenic substances. These substances include protons, bradykinin, endothelins, prostaglandins, proteases, and growth factors such as nerve growth factor (NGF) and vascular endothelial growth factor (VEGF) ${ }^{1-4}$ that can interact with peripheral nerve and cause increased hypersensitivity and excitability of nociceptive neurons ${ }^{3,5,6}$. NGF and VEGF also induce outgrowth of pain-conducting nerve fibers in cancer affected areas ${ }^{3,4}$. Despite current focus on cancer-produced pronocicepetive mediators ${ }^{7}$, early-stage cancers before metastasis to bone tissues are often not painful ${ }^{2,8}$ and pain in melanoma is not common prior to metastasis ${ }^{9}$. It is conceivable that different cancers and even the same cancers at different growth stages may produce different pain mediators that can differentially regulate pain sensitivity via positive or negative modulation ${ }^{10}$.

Mounting evidences suggests that cancers, such as melanoma express the checkpoint inhibitory protein PD-L1, which can suppress T cell function and induce immune tolerance via its receptor PD-1 ${ }^{11-14}$. Emerging immune therapy such as anti-PD1 and anti-PD-L1 treatment has shown success in treating cancers such as melanoma ${ }^{8,15,16}$, as well as lymphoma, lung cancer, ovarian cancer, and head and neck cancers ${ }^{17-19}$. However, it is unclear whether and how the PD-L1/PD-1 pathway can regulate pain sensitivity via nonimmune modulation such as neuronal modulation. It is increasingly appreciated that primary nociceptive neurons (nociceptors) share similarities with immune cells and can both listen and talk to immune cells ${ }^{10,20,21}$. Nociceptors not only respond to immune mediators such as cytokines and chemokines and bacterial infection ${ }^{22}$ but also produce cytokines and chemokines and express Toll-like receptors (TLRs), key regulators of immunity ${ }^{20,23-25}$. In primary sensory neurons, TLRs rapidly regulate pain sensitivity via interacting with ion channels ${ }^{10,26}$. It remains unclear if nociceptive neurons express functional PD-1 receptor, an important immune regulator, in mouse and human dorsal root ganglion (DRG).

In this study, we assessed the expression and function of PD-1 in primary sensory neurons of mouse and human DRG. We find that activation of PD-1 by PD-L1 potently suppresses neuronal activities in mouse and human nociceptive neurons. Moreover, PD-L1 inhibits acute baseline pain and inflammatory pain and chronic neuropathic pain after nerve injury. In addition to malignant melanoma tissue, endogenous PD-L1 can be detected in normal neural tissues including spinal cord, DRG, nerve and skin. We also discovered that PD-L1 potently suppresses spinal cord synaptic transmission in the pain circuit as a unique neuromodulator. Finally, PD-L1 masks pain in melanoma, and conversely, blocking PD-L1 or PD-1 elicits spontaneous pain and allodynia in melanoma-bearing mice.

\section{RESULTS}

\section{PD-L1 inhibits acute inflammatory pain and increases pain threshold in naïve animals}

As a first step to address a role of PD-L1 in acute pain modulation, we examined the effects of PD-L1 in an acute inflammatory pain model. Intraplantar (i.pl) injection of formalin (5\%) 
induced typical bi-phasic inflammatory pain as previously reported ${ }^{27}$, but the $2^{\text {nd }}$-phase pain (10-45 min) was substantially inhibited by PD-L1 pre-treatment (i.pl., 1-10 $\mu \mathrm{g}, P<0.05$, One-Way ANOVA), in a dose-dependent manner (Fig. 1a). At high doses (5 and $10 \mu \mathrm{g}$ ), PDL1 also caused a mild inhibition of the $1^{\text {st }}$-phase pain (Fig. 1a).

Next, we tested if PD-L1 would also alter pain threshold in naïve mice. Von Frey test revealed a significant increase in paw withdrawal threshold after i.pl. injection of PD-L1 (5 $\mu \mathrm{g} \approx 0.1 \mathrm{nmol}, P<0.05$, Two-Way ANOVA). The threshold increase was rapid and evident at $30 \mathrm{~min}$ and maintained for $3 \mathrm{~h}$ after the injection (Fig. 1b). Since PD-L1 (CD274) is a chimera protein fused with human IgG at the C-terminal, we used human IgG as an inactive control (http://www.abcam.com/recombinant-mouse-pd-11-protein-fc-chimera-activeab180058.html). Notably, this human IgG had no effect on the pain threshold (Fig. 1b).

\section{PD-L1 is an endogenous pain inhibitor and alters basal pain thresholds via PD-1}

PD-L1 is produced by malignant tissues and serves as a predictive biomarker in cancer immunotherapy ${ }^{28}$. As expected, mouse B16 melanoma tissue has high expression levels of PD-L1 ( $\approx 450 \mathrm{ng} / \mathrm{mg}$ tissue, Fig. 2a), as evaluated by ELISA analysis. PD-L1 was also secreted in cultured medium of melanoma cells (Supplementary Fig. 1a). To determine if normal tissues also produce PD-L1, we compared PD-L1 contents in non-neural and neural tissues. Non-neural tissues, such as liver, spleen, and kidney have high levels of PD-L1 $(\approx 70-90 \mathrm{ng} / \mathrm{mg}$ tissue, Fig. 2a). Interestingly, neural tissues, including brain, spinal cord, and dorsal root ganglia (DRG) contact PD-L1 at levels around $50 \mathrm{ng} / \mathrm{mg}$ tissue (Fig. 2a). Furthermore, PD-L1 was detected in the sciatic nerve and hindpaw skin tissues (Fig. 2a), which contain pain-sensing nerve fibers. These results suggest that PD-L1 is broadly synthesized by neural and skin tissue. In agreement, in situ hybridization revealed Pdl1 mRNA expression in mouse DRG neurons (Supplementary Fig. 1b,c).

To determine a role of endogenous PD-L1, produced by non-malignant tissues, in pain regulation, we tested mechanical pain after pharmacological blockade of either PD-L1 or PD-1 in naïve mice. Neutralization of hindpaw PD-L1 by i.pl. injection of soluble PD-1 (sPD-1, $5 \mu \mathrm{g} \approx 0.1 \mathrm{nmol}$ ) induced a transient mechanical allodynia for $3 \mathrm{~h}$ (Fig. 2b), without causing spontaneous pain (Supplementary Fig. 2a). Blockade of PD-1 with a mouse antiPD-1 antibody, RMP1-14 (5 $\mu \mathrm{g}, \approx 0.1 \mathrm{nmol}$, i.pl.), also induced mechanical allodynia for $3 \mathrm{~h}$ (Fig. 2b).

Nivolumab is a FDA-approved fully humanized IgG4 monoclonal antibody, which selectively targets PD- ${ }^{29}$ and has shown great success in treating melanoma, lymphoma, and lung cancer ${ }^{17,29,30}$. Of note Nivolumab $(10 \mu \mathrm{g} \approx 0.07 \mathrm{nmol}$, i.pl.) but not control human $\mathrm{IgG}$, induced marked mechanical allodynia for $5 \mathrm{~h}$ (Fig. 2b). PD-L1's analgesic effects were blocked by both RMP-14 and Nivolumab (Supplementary Fig. 2b), suggesting that PD-L1 inhibits pain via PD-1. As a human antibody Nivolumab showed cross-activity in mouse tissue and binding on DRG neurons and sciatic nerve fibers in wild-type (WT) mice, but this binding was absent in $P d 1$ knockout mice (KO, $P d 1^{-/-}$, Supplementary Fig. 2c).

Next we tested baseline pain and PD-L1-induced analgesia in $P d 1^{-1-}$ mice with and without PD-L1 treatment. Interestingly, baseline pain sensitivity increased in naive $\mathrm{PdI}^{-/-}$mice. 
Compared with WT mice $P d 1^{-/-}$mice displayed mechanical and thermal hypersensitivity, by showing decreased mechanical and thermal pain thresholds in von Frey test and hot plate test (Fig. 2c,d). This result indicates an essential role of PD-1 in regulating basal pain sensitivity. As expected, both PD-L1-induced analgesic effect and RMP1-14-induced hyperalgesic effect were abolished in $\mathrm{Pd}^{-/-}$mice (Supplementary Fig. 3a,b). Notably, $P d 1^{-1-}$ mice showed no developmental defects in sensory neurons and their innervations. The central innervations of primary afferents in the spinal cord dorsal horn are comparable in WT and KO mice (Supplementary 4). The distribution patterns of primary sensory neurons, including small-sized nociceptive neurons $\left(\mathrm{CGRP}^{+}\right.$peptidergic neurons and $\mathrm{IB}^{+}$ non-peptidergic neurons) and large-sized A-fiber DRG neurons ( $\mathrm{NF}^{200^{+}}$), as well as the total population of sensory neurons are also unaltered in DRG tissues of KO mice (Supplementary Fig. 5). Taken together, these findings in WT and $\mathrm{PdI}^{-1-}$ mice strongly suggest that 1) PD-L1 is an endogenous inhibitor of pain, 2) PD-L1 produces analgesia via PD-1, and 3) altered pain sensitivity in $P d 1^{-/-}$mice is not a result of developmental defects in sensory neurons.

\section{PD-1 receptor is expressed by primary sensory neurons in mouse DRGs}

To determine peripheral mechanisms by which PD-L1 modulates pain, we examined Pd1 mRNA and PD-1 protein expression in mouse DRG neurons. In situ hybridization showed Pd1 mRNA expression in majority of DRG neurons with various sizes (Fig. 3a,b). This expression was lost in $\mathrm{PdI}^{-1-}$ mice (Fig. 3c) and in DRG sections treated with sense control probe (Fig. 3d), confirming the specificity of $P d 1 \mathrm{mRNA}$ expression. Immunohistochemistry reveled PD-1 immunoreactivity (IR) in majority of DRG neurons (Fig. 3e). The specificity of the PD-1 antibody was validated by loss of PD-1 immunostaining in DRG neurons of $\mathrm{Pd}^{-1-}$ mice (Fig. 3e) and further confirmed by absence of staining in WT DRG after coincubation of the antibody with a blocking peptide (Fig. 3e). Size frequency analysis showed a broad expression of PD-1 by DRG neurons with small, medium, and large sizes (Fig. 3f). Double staining confirmed PD-1 expression in both large-diameter A-fiber neurons $\left(\mathrm{NF}_{200^{+}}\right.$) and small-diameter C-fiber neurons (NF200-, Fig. 3g). PD-1-IR was present in $\mathrm{NF}_{200^{+}}$and $\mathrm{NF}_{200^{-}}$axons in the sciatic nerve, indicating an axonal transport of PD-1 from DRG cell bodies to peripheral axons (Fig. 3h). PD-1-IR axons co-express CGRP, a marker for nociceptive peptidergic neurons (Fig. 3i). Together, these results demonstrate that primary sensory neurons, including nociceptors and their axons, express PD-1, providing a neuronal base for PD-1 modulation of pain.

\section{PD-L1 suppresses nociceptive neuron activity in mouse DRGs via PD-1}

Activation and sensitization of nociceptive sensory neurons (nociceptors) often produces pain and pain hypersensitivity ${ }^{31-33}$. We postulated that PD-L1/PD-1 inhibits pain via direct modulation of nociceptor activity. We employed patch clamp recordings to evaluate excitability in dissociated small-diameter neurons ( $<25 \mu \mathrm{m}$, presumably nociceptors) in mouse DRGs. Notably, PD-L1, at a very low concentration $(10 \mathrm{ng} / \mathrm{ml} \approx 0.2 \mathrm{nM})$, evoked a potent and immediate inhibition of action potential induced by current injection and further increased rheobase, a minimum current to induce action potential (Fig. 4a). PD-L1 also induced hyperpolarization of the resting membrane potential (RMP) in DRG neurons (Fig. 4b). These effects of PD-L1 on action potentials and RMPs were abrogated in $\mathrm{PdI}^{-{ }^{-}}$mice, 
indicating that PD-L1 modulates neuronal excitability through PD-1 (Fig. 4a,b).

Furthermore, Pd1-deficient nociceptive neurons displayed increased RMP and firing frequency of action potentials (Fig. 4c,d), suggesting that the intrinsic excitability of nociceptors is enhanced in $P d 1$ mutant mice.

To further assess the contribution of endogenous PD-L1 and PD-1 to neuronal excitability in WT neurons, we employed pharmacological approaches in a whole mount DRG preparation. Compared to dissociated DRG neurons, whole mount DRG preparation has advantage of retaining extracellular PD-L1. Neutralization of PD-L1 with sPD-1 $(30 \mathrm{ng} / \mathrm{ml} \approx 0.6 \mathrm{nM})$ increased the firing rate of action potentials in small-diameter DRG neurons (Fig. 4e). Blocking the function of PD-1 with Nivolumab, but not the control $\operatorname{IgG}(300 \mathrm{ng} / \mathrm{ml} \approx 2 \mathrm{nM})$, also increased the firing rate (Fig. 4f). Together, both gain-of-function and loss-of-function approaches demonstrate a critical role of PD-L1/PD-1 in regulating excitability of nociceptive neurons.

\section{PD-L1 inhibits neuronal hyperexcitability and neuropathic pain after nerve injury}

Hyperexcitability of primary sensory neurons after nerve injury has been strongly implicated in chronic pain ${ }^{31,33-35}$. We used mount mouse DRG preparation to examine hyperexcitability in small-sized nociceptive neurons after chronic nerve constriction (CCI). As expected, nociceptive neurons fired more action potentials after CCI (Fig. 5a). Notably, nerve injury-induced hyperexcitability (i.e. increased firing rate of action potentials) of DRG neurons was dose-dependently suppressed by PD-L1 (1-10 ng/ml $\approx 0.02-0.2 \mathrm{nM}$, Fig. 5a,b).

The central axons of nociceptive neurons terminate in the spinal cord dorsal horn to form first-order synapses in the pain pathway ${ }^{33}$. PD-L1 in DRG neurons could be transported to central axon terminals to modulate spinal cord synaptic transmission and nociception. To test this hypothesis, we examined the effects of intrathecal (i.t.) injection of PD-L1 on CCIinduced neuropathic pain in mice. PD-L1 reduced the CCI-induced mechanical allodynia at a low dose (100 ng, Fig. 5c). PD-L1 also significantly reduced CCI-induced heat hyperalgesia at a high dose $(1 \mu \mathrm{g}, P<0.05$, Two-Way ANOVA, Fig. $5 \mathrm{~d})$. Randall-Selitto test further revealed that intrathecal PD-L1 increased paw withdrawal threshold in naïve mice (Fig. 5e).

\section{PD-L1 inhibits synaptic transmission and injury-induced neuronal hyperactivities in the spinal cord}

Patch clamp recordings in spinal cord slices showed that superfusion of PD-L1 rapidly (within $1 \mathrm{~min}$ ) reduced the frequency and amplitude of spontaneous EPSCs (sEPSCs) in lamina IIo neurons (Supplementary Fig. 6a). These interneurons form a nociceptive circuit with C-fiber afferents and projection neurons ${ }^{36,37}$. By sharp contrast, exposure of spinal cord slices to sPD-1 (PD-L1 neutralization) and Nivolumab (PD-1 blockade) increased sEPSC frequency in lamina IIo neurons (Supplementary Fig. 6b,c). As expected, PD-L1's inhibition of sEPSC frequency was blocked by Nivolumab (Supplementary Fig. 6d). Thus, PD-L1/ PD-1 also has an active role in modulating spinal nociceptive transmission.

Next, we tested the central effects of PD-L1 in a bone cancer model in rats ${ }^{38}$. PD-L1, given two weeks after tumor cell inoculation via i.t. route, reduced bone cancer-induced 
mechanical allodynia ( $P<0.05$, Two-Way ANOVA, Supplementary Fig. 7a). Moreover, bone cancer-induced hyperexcitability of wide dynamic range (WDR) neurons in dorsal horn neurons was suppressed by PD-L1 (Supplementary Fig. 7b,c), whereas Nivolumab enhanced activities of WDR neurons (Supplementary Fig. 7d,e). Taken together, our data suggest that PD-L1 is a neuromodulator in both the peripheral and central nervous system; and in the spinal cord PD-L1 regulates acute and chronic pain by suppressing nociceptive synaptic transmission and injury-induced neuronal plasticity in dorsal horn neurons via PD-1 receptor.

\section{PD-L1 modulates sodium currents and TREK2 potassium channels via SHP-1}

How does PD-L1 modulate neuronal excitability? Activation of PD-1 by PD-L1 recruits the tyrosine phosphatases SHP-1/SHP-2 (Src homology region 2 domain-containing phosphatase-1 and 2) to mediate PD-L1's biological actions in immune cells ${ }^{13,39}$. Immunohistochemistry shows that PD-L1 is sufficient to activate SHP-1 in vivo after i.t. injection, leading to increased phosphorylation of SHP-1 (pSHP-1) in mouse DRG neurons (Fig. 6a). In agreement, pSHP-1 was co-localized with Pd1 mRNA in DRG neurons (Supplementary Fig. 8a,b). Moreover, PD-L1-induced SHP-1 phosphorylation was blocked by SHP-1 inhibitor sodium stibogluconate (SSG) in dissociated DRG neurons (Supplementary Fig. 8c). Intraplantar administration of PD-L1 induced analgesia by reducing paw withdrawal frequency in naïve animals; but this analgesic effect of PD-L1 was abolished by i.pl. SSG (Fig. 6b). Thus, SHP-1 is not only a downstream signaling event following PD-1 activation in DRG neurons but also contributes to PD-L1-evoked analgesia.

Given an important role of sodium channels in generating action potentials and pain ${ }^{40}$, we examined the effects of PD-L1 on transient sodium currents in mouse DRG neurons with small diameters. PD-L1 perfusion $(10 \mathrm{ng} / \mathrm{ml})$ caused a gradual and persistent inhibition of transient sodium currents (Fig. 6c). Moreover, PD-L1-induced inhibition of sodium currents was partially blocked by the SHP inhibitor SSG (Fig. 6c), supporting an involvement of SHP.

Two-pore $\mathrm{K}^{+}$channel TREK2 plays a major role in regulating RMP in DRG nociceptive neurons of rats ${ }^{41}$. TREK2 also expressed in mouse DRG neurons (Supplementary Fig. 9a). We assessed if PD-L1 would modulate TREK2 activity in heterologous CHO cells. PD-L1 caused hyperpolarization of RMP ( $\triangle \mathrm{RMP} \approx 8 \mathrm{mV})$ in CHO cells co-expressing PD-1 (encoded by $P D 1$ or $P D C D 1$ ) and TREK2 (encoded by $K C N K 10$ ), but this change was blocked by SSG (Fig. 6d). PD-L1 also potentiated TREK2-induced currents and produced a negative shift in reversal potential $\left(\mathrm{E}_{\mathrm{rev}}\right)$ in PD-1/TREK2 co-expressing CHO cells; and both were abolished by SSG (Fig. 6e). However, PD-L1 alone was insufficient to alter the voltage-ramp currents and $\mathrm{E}_{\mathrm{rev}}$ in $\mathrm{CHO}$ cells expressing either TREK2 or PD-1 (Supplementary Fig. 9b). Collectively, activation of PD-1 by PD-L1 might modulate neuronal excitability by suppressing the function of sodium channels and enhancing the function of potassium channels (TREK2) via SHP-1 (Supplementary Fig. 9c). 


\section{Human DRG neurons express functional PD-1}

A translational gap from rodents to humans was blamed for many failures in developing pain therapeutics ${ }^{42,43}$. To this end, we examined the PD-1 expression and function in human DRG neurons from non-diseased donors, as shown in our previous studies ${ }^{25,44}$. PD-1 IR was observed on cell surface of human DRG neurons with small and large sizes as well as in human spinal nerve axons (Fig. 7a, Supplementary Fig. 10a,b). This staining in human DRG and nerve sections was abolished by the immunizing blocking peptide (Supplementary Fig. 10a,b). Both NF200 positive and negative axons of human spinal nerve expressed PD-1 (Supplementary Fig. 10c).

Importantly, PD-1 receptor is functional in human DRG neurons: incubation of dissociated small-diameter nociceptive neurons $(30-50 \mu \mathrm{m})$ with PD-L1 directly altered neuronal activities. At an concentration $(10 \mathrm{ng} / \mathrm{ml})$ that is effective in suppressing mouse nociceptive neuron activity (Fig. 4a,b), PD-L1 markedly inhibited the firing frequency of action potentials and further increased the threshold for action potential induction (rheobase) in human DRG neurons (Fig. 7b,c). PD-L1 also caused hyperpolarization of human nociceptive neurons by decreasing RMP (Fig. 7d). Additionally, PD-L1 perfusion (10 ng/ml) caused a gradual and persistent inhibition of transient sodium currents in human DRG neurons (Fig. 7e). Notably, PD-L1-induced inhibition of sodium currents was partially blocked by SSG in mouse DRG neurons (Fig. 6c) but completely blocked by SSG in human DRG neurons (Fig. 7e), supporting an important role of SHP in regulating PD-L1 signaling in human sensory neurons.

\section{PD-L1 and PD-1 mask spontaneous pain and allodynia in a mouse melanoma model}

Given the high expression of PD-L1 in melanoma (Fig. 2a), we examined the contribution of PD-L1 and PD-1 to altered pain sensitivity in a mouse model of melanoma. Intraplantar injection of mouse melanoma cells $\left(5 \times 10^{5}\right.$ cells in $\left.20 \mu \mathrm{l}\right)$ into C57BL/6 mice lead to timedependent tumor growth in a hindpaw, showing a 3-fold increase in paw volume 4 weeks after melanoma cell implantation (MCI-4w, Fig. 8a). Melanoma-bearing mice also exhibited increased PD-L1 levels in serum at MCI-4w (Fig. 8b). Despite profound tumor growth, we did not observe cardinal features of cancer pain, including mechanical allodynia and spontaneous pain (licking/flinching the tumor-bearing paw) in tumor-bearing hind paws (Fig. 8c, d).

Next, we tested the hypothesis that pain after melanoma could be masked by upregulated PD-L1 function. We employed several pharmacological approaches to block PD-L1/PD-1 signaling. Strikingly, local neutralization of PD-L1, by i.pl. injection of soluble PD-1 (sPD-1, $5 \mu \mathrm{g}, \mathrm{MCI}-4 \mathrm{w}$ ), elicited marked spontaneous pain (Fig. 8e). The onset of spontaneous pain was very rapid: mice displayed licking/flinching behavior in melanomabearing paws within 10-30 min after the injection. This spontaneous pain was also phasic, showing a peak every hour for the first $3 \mathrm{~h}$ (Supplementary Fig. 11a, Supplementary video 1-2). The same sPD-1 treatment also induced mechanical allodynia (Supplementary Fig. 11b). Conditioned place preference (CPP) is an operant measurement of ongoing pain ${ }^{35}$. Using a two-chamber test, we found that i.pl. sPD-1 treatment also resulted in marked CPP (Fig. 8f). 
Given an important role of PD-L1 in regulating the function of immune system, we also investigated the effects of sPD-1 treatment on T cell and inflammatory markers in the ipsilateral hindpaw skin surrounding melanoma and control skin in the contralateral paw. To correlate the changes of these immune markers with pain, we collected skin tissues in the acute phase, $3 \mathrm{~h}$ after the sPD-1 treatment when robust allodynia and spontaneous pain developed. MCI resulted in increased mRNA levels of T cell markers (CD2, CD3), macrophage marker (CD68), and inflammatory cytokine markers (TNF, IL-1B, IL-6, IFNG, CCL2) in the ipsilateral skin, compared with the contralateral skin (Supplementary Fig. 12). However, the mRNA levels of these immune/inflammatory markers did not alter after the sPD-1 treatment (Supplementary Fig. 12). This result further indicates that sPD-1 induces pain via non-immune modulation, at least in the acute phase (i.e. first $3 \mathrm{~h}$ ).

To further test a peripheral and neuronal role of PD-1 in regulating pain in melanoma, we employed a gene therapy method we recently established ${ }^{27}$ in which small interfering RNA (siRNA) was used to knockdown PD-1 expression specifically in DRG neurons. This method allows siRNA uptake by DRG sensory neurons via axonal retrograde transport of siRNA ${ }^{27}$. Peri-sciatic injection of PD-1-targeting siRNA at MCI-4w induced marked and persistent mechanical allodynia for $>4$ days (Fig. $8 \mathrm{~g}$ ) and further evoked spontaneous pain in melanoma-bearing mice (Fig. 8h). Compared to non-targeting control siRNA, this Pd1targeitng siRNA partly but significantly reduced PD-1 expression in mouse DRG and sciatic nerve but not in spinal cord tissues ( $P<0.05$, Supplementary Fig. 13). Thus, PD-1 expressed by DRG neurons could be sufficient to mask cancer pain.

Finally, we evaluated if anti-PD-1 antibodies would also unmask pain as SPD-1 and Pd1 siRNA in the melanoma model. Intravenous injection of Nivolumab, but not the control human IgG4 (3-10 mg/kg), caused rapid, persistent, and dose-dependent mechanical allodynia and also elicited marked spontaneous pain (Fig. 8i,j). Furthermore, RMP1-14 (10 $\mathrm{mg} / \mathrm{kg}$, i.v.), a mouse anti-PD-1 antibody, evoked remarkable spontaneous pain and mechanical allodynia (Supplementary Fig. 14a,b). In vivo recordings in the mouse sciatic nerve showed that i.v. Nivolumab significantly increased spontaneous discharges in nerve fibers (Fig. 8k,1), indicating that anti-PD-1 treatment can unmask pain by increasing the excitability of primary afferent fibers. Moreover, local injection of Nivolumab via intrathecal or intraplantar route each evoked mechanical allodynia in melanoma-bearing mice (Supplementary Fig. 14c,d). Blocking the downstream signaling of PD-1 with SHP-1 inhibitor SSG also elicited spontaneous pain (Supplementary Fig. 14e). Together, these findings suggest that PD-L1 can mask pain in non-metastatic melanoma via PD-1 and SHP (Supplementary Fig. 14f).

\section{DISCUSSION}

The prevailing view in the field is cancers secrete pronociceptive mediators to activate or sensitize and primary afferent neurons in the cancer microenvironment. This microenvironment contains growth factors such as NGF and VEGF that cause sprouting of pain-sensing afferent fibers ${ }^{3,4}$. In this study, we have demonstrated that cancers also produce the anti-nociceptive mediator PD-L1 to suppress pain. In particular, we reveal PD-L1 as a previously unrecognized endogenous inhibitor of pain: PD-L1 is produce not only by 
melanoma but also by non-malignant tissues such as skin, DRG, and spinal cord. In naïve mice, exogenous application of PD-L1 induced analgesia, whereas blockade of endogenous PD-L1 and PD-1 signaling via sPD-L1, PD-1 antibodies, or Pd1 deletion resulted in hyperalgesia. PD-L1 increased pain threshold via PD-1 receptor, because the analgesic effect of PD-L1 was completely lost in mice lacking Pd1. In addition to physiological pain in naïve animals, PD-L1 potently suppressed formalin-induced acute inflammatory pain.

Furthermore, PD-L1 effectively reduced chronic pain including nerve injury-induced neuropathic pain and bone cancer pain in rodents via both peripheral and central actions.

We have also demonstrated that PD-L1 is a novel neuromodulator that modulates neuronal excitability in mouse and human DRGs of the peripheral nervous system and synaptic transmission in the spinal cord of the central nervous system, through activation of PD-1 receptor. It is generally believed that PD-1 is expressed by immune cells such as T cells ${ }^{13}$. However, non-immune cells such as melanoma cells also express PD- ${ }^{45}$. Our analyses using immunohistochemistry, in situ hybridization, and electrophysiology in dissociated DRG neurons clearly demonstrate the presence of anatomical and functional PD-1 receptor in mouse and human DRG neurons.

Mechanistically, our results show that activation of PD-1 by PD-L1 inhibited action potential induction and suppressed transient sodium currents in mouse and human DRG neurons. PDL1 also regulated RMPs and caused hyperpolarization, via PD-1/SHP activation and subsequent activation of two-pore $\mathrm{K}^{+}$channel TREK2. Furthermore, PD-L1 was present in spinal cord tissue and bath application of PD-L1 suppressed excitatory synaptic transmission (sEPSC) in lamina IIo neurons in the spinal cord pain circuit. PD-L1 also inhibited bone cancer-induced hyperexcitability in spinal WDR neurons. These results strongly suggest that as a neuromodulator PD-L1 modulates pain sensitivity via both peripheral and central mechanisms. Because PD-L1 affects both the frequency and amplitude of sEPSCs in spinal cord slices (Supplementary Fig. 6), PD-1 may also be present in postsynaptic neurons in the spinal cord and brain. Future study is necessary to investigate signaling mechanisms by which PD-L1/PD-1 regulates synaptic transmission and synaptic plasticity in the spinal cord and brain. Given an important role of immune cells in chronic pain sensitization ${ }^{10,46}$, it is conceivable that PD-L1 could control chronic pain by suppressing T-cell activation and proinflammatory responses ${ }^{47}$. However, given the time scale of neuromodulation (minutes and hours), the rapid changes in pain behavior after the manipulations of the PD-L1/ PD-1/SHP pathway are likely to be mediated by neuronal activation. Growing evidence supports an important role of glial cells such as microglia and astrocytes in the pathogenesis of pain $10,48-50$. We should not exclude the possibility that PD-L1/PD-1 may also regulate glial signaling in persistent pain.

It is noteworthy that PD-L1 suppressed pathological pain not only in models of inflammatory, neuropathic, and bone cancer pain but also in a melanoma model, which exhibited high PD-L1 levels in circulation. We provided several lines of pharmacological and behavioral evidence to demonstrate a critical role of the PD-L1/PD-1 axis in masking pain in melanoma-bearing mice. First, inoculation of B16-melanoma cells resulted in robust melanoma growth but not spontaneous pain and mechanical allodynia. Second, intraplantar neutralization of PD-L1 with soluble PD-1 induced spontaneous pain, ongoing pain (CPP), 
and mechanical allodynia; and furthermore, systemic or local injection of either human antiPD-1 antibody (Nivolumab) or mouse anti-PD-1 antibody (RMP1-14), or siRNA knockdown of PD-1 expression in DRGs each induced robust pain symptoms in melanoma-bearing hindpaw. Finally, inhibition of SHP also evoked spontaneous pain. It is of great interest to investigate whether PD-L1 can still mask pain after melanoma metastasis.

What is the biological significance of PD-L1 in suppressing the function of both immune system and nociceptive system? Because these two systems are important for host defense $^{20,21}$, it is conceivable that tumor can shut off both defense systems via PD-L1 secretion for optimal host invasion and cancer growth. Emerging immune therapies with anti-PD1 and anti-PD-L1 antibodies have shown efficacy in treating cancers such as melanoma ${ }^{8,15,16}$. Our findings suggest the importance of examining the pain caused by individual tumor sites in patients with melanoma and other malignancies before, after, and during immune therapies. On the other hand, it is also of great interest to identify novel pain inhibitors produced by cancer cells, which will open a new avenue to developing future pain medicine. Given the high potency of PD-L1 in suppressing activities of human nociceptive neurons, local targeting of PD-L1/PD-1 signaling in sensory neurons may lead to the development of novel analgesics.

\section{ONLINE METHODS}

\section{Reagents}

Mouse PD-1 (Catalog: 1021-PD-100) and Rat IgG2A Isotype control (Catalog: MAB006) was obtained from R\&D. Mouse PD-L1 (Catalog: ab180058) and human IgG4 (Catalog: ab90286) were purchased from Abcam. Nivolumab (OPDIVO ${ }^{\circledR}$ ), a humanized anti-PD-1 antibody, was purchased from Bristol-Myers Squibb. Anti-mouse PD-1 antibody RMP1-14 (Catalog: BE0146) was from Bio X Cell. Mouse Pd1-targeting siRNA (Catalog: L-040330-01-0005) and non-targeting siRNA (Catalog: D-0018100-01-20) were purchased from Thermo Scientific Dharmacon. RVG peptide was synthetized by Invitrogen and mixed with siRNA to increase neuronal uptake of siRNA by axons in the sciatic nerve ${ }^{27}$. SHP-1 inhibitor sodium stibogluconate (SSG) was from Calbiochem (Catalog: 567565). PD1/ PDCD1 cDNA construct (SC117011, NM_005018) and TREK2/KCNK10 cDNA construct (SC110477, NM_021161) were purchased from Origene Technologies.

\section{Animals}

Adult mice (males, $8-10$ weeks) were used for behavioral and biochemical studies. Pd1 knockout mice with C57BL/6 background were purchased from the Jackson laboratory (Stock No: 021157) and maintained at Duke animal facility. Young mice (5-7 weeks of both sexes) were used for electrophysiological studies in DRG neurons. All the mouse procedures were approved by the Institutional Animal Care \& Use Committee of Duke University. For bone cancer pain experiment, adult Wistar rats (females, 8 weeks) were obtained from Shanghai Experimental Animal Center of Chinese Academy of Sciences and the rat experiments were approved by the Animal Care and Use Committee of Fudan University. All animals were housed under a 12-hour light/dark cycle with food and water available ad libitum. No statistical method was used to predetermine sample size. No randomization was 
applied to the animal experiments. Sample sizes were estimated based on our previous studies for similar types of behavioral, biochemical, and electrophysiological analyses ${ }^{25,27,35}$. Two to five mice or rats were housed in each cage. Animal experiments were conducted in accordance with the National Institutes of Health Guide for the Care and Use of Laboratory Animals. The numbers of mice and rats used in different experiments were summarized in Supplementary Fig. 15.

\section{Culture of murine melanoma cells}

Murine melanoma cell line B16-F10 was obtained from ATCC (ATCC ${ }^{\circledR}$ CRL-6475, Rockville, Maryland). Melanoma cells were grown in Dulbecco's modified Eagle medium containing $4500 \mathrm{mg} / \mathrm{l}$ glucose, $100 \mathrm{mg} / \mathrm{l}$ penicillin, $100 \mathrm{mg} / \mathrm{l}$ streptomycin, and supplemented with $10 \%$ fetal bovine serum in $5 \% \mathrm{CO}_{2} / 95 \%$ air at $37{ }^{\circ} \mathrm{C}$. Cells were collected for experiments following enzymatic digestion with trypsin.

\section{Mouse and rat models of cancer and pain}

We produced the following rodent models of pain.

Mouse model of melanoma-Murine B16-F10 melanoma cells ( $5 \times 10^{5}$ cells/20 $\mu$ l, suspended in PBS) were subcutaneously injected into the plantar region of a left hindpaw of mouse.

Mouse model of inflammatory pain-Acute inflammatory pain was induced by intraplantar injection of $20 \mu \mathrm{l}$ diluted formalin (5\%).

Mouse model of neuropathic pain-Chronic constriction injury (CCI) model of neuropathic pain was produced under isoflurane anesthesia ${ }^{35}$. After the left sciatic nerve was exposed, three ligatures (7-0 prolene) were placed around the nerve proximal to the trifurcation with one millimeter between each ligature. The ligatures were loosely tied until a short flick of the ipsilateral hind limb was observed. Animals in the sham group received surgery identical to those described but without nerve ligation.

Rat bone cancer pain model-Tumor cells were extracted from the ascetic fluid of rats that received Walker 256 rat mammary gland carcinoma cells, and suspension of $1 \times 10^{8} / \mathrm{ml}$ tumor cells in PBS was prepared. The inoculation was performed as previously described ${ }^{38}$. Briefly, rats were anesthetized with sodium pentobarbital $(50 \mathrm{mg} / \mathrm{kg}$, intraperitoneal). The right leg was shaved, and the skin was disinfected with iodine tincture and $75 \%$ ethanol. A 22-gauge needle was inserted at the site of the intercondylar eminence of the right tibia and was then replaced with a $10 \mu \mathrm{l}$ microinjection syringe containing a $4 \mu \mathrm{l}$ suspension of tumor cells $\left(4 \times 10^{5}\right)$. The contents of the syringe were slowly injected into the tibia cavity. To prevent leakage of cells outside the bone, the injection site was sealed with bone wax. For the sham group (control), $4 \mu \mathrm{l}$ of PBS was injected instead of carcinoma cells into the tibia. At the end of the experiment, radiological, postmortem, and histological evaluations were performed. Rats that showed no obvious tumor growth and bone destruction after inoculation of tumor cells were excluded from the experiments. 


\section{Drug injection}

For intravenous injection, anti-PD-1 antibody (Nivolumab, 3 or $10 \mathrm{mg} / \mathrm{kg}$ or RMP1-14, 10 $\mathrm{mg} / \mathrm{kg}$ in $100 \mu \mathrm{l}$ PBS) or control antibody (human IgG4 or rat IgG2A) was administered into the tail vein of mouse. For local intraplantar injection, drugs (20 $\mu \mathrm{l} \mathrm{PBS)} \mathrm{were} \mathrm{injected} \mathrm{using}$ a Hamilton microsyringe (Hamilton) with a 30-gauge needle. For intrathecal injection, spinal cord puncture was made with a 30-G needle between the L5 and L6 level to deliver reagents $(10 \mu \mathrm{l})$ to the cerebral spinal fluid ${ }^{35}$. For peri-sciatic injection, a mixture of $2 \mu \mathrm{g}$ siRNA and $1.5 \mu \mathrm{g}$ of transfection reagent (Chimeric Rabies Virus Glycoprotein Fragment, RVG-9R ${ }^{27}$ ) in $6 \mu \mathrm{D} 5 \mathrm{~W}$ ( $5 \%$ dextrose in water) was injected with a $30-\mathrm{G}$ needle under the mesoneurium of the left sciatic nerve at mid-thigh level. Care was taken to avoid solution entry into the epineurium of the sciatic nerve.

\section{In situ hybridization}

We used probes directed against mouse PdI1 (NM_021893) and Pdcd1 (NM_008798) designed by Advanced Cell Diagnostics and the RNAscope multiplex fluorescent assay according to the manufacturer's instructions. Pre-hybridization, hybridization and washing were performed according to standard methods ${ }^{25}$.

\section{Immunohistochemistry in mouse and human tissues and quantification}

After appropriate survival times, mice were deeply anesthetized with isoflurane and perfused through the ascending aorta with PBS, followed by $4 \%$ paraformaldehyde. After the perfusion, the L4-L5 spinal cord segments, L4-L5 DRGs, sciatic nerves, and melanoma tissues were removed and postfixed in the same fixative overnight. Fresh human DRGs (L4L5) of 4 non-diseased donors from NDRI (National Disease Research Interchange) ${ }^{25}$ and the attached spinal nerves were immediately fixed upon delivery in fresh $4 \%$ paraformaldehyde overnight. Spinal cord, DRG, and nerve tissue sections (10 or $14 \mu \mathrm{m}$ ) and free-floating spinal cord and skin sections $(30 \mu \mathrm{m})$ were cut in a cryostat. The sections were blocked with $2 \%$ goat or donkey serum for $1 \mathrm{~h}$ at room temperature and then incubated overnight at $4{ }^{\circ} \mathrm{C}$ with the following primary antibodies: anti-PD-1 (rabbit, 1:500, Sigma, Catalog: PRS4065), anti-phosphorylated SHP-1 (pSHP-1, rabbit, 1:500, Abcam, Catalog: ab51171), anti-NeuN (mouse, 1:1000, Millipore, Catalog: MAB377), anti-NF200 (mouse, 1:1000, Sigma, Catalog: N0142), anti-TREK2 (rabbit, 1:200, Alomone labs, Catalog: APC-055), and antiCGRP (goat, 1:500, Abcam, Catalog: ab36001) antibodies. After washing, the sections were incubated with cyanine 3(Cy3)- and/or FITC-conjugated secondary antibodies (1:400; Jackson ImmunoResearch) for $2 \mathrm{~h}$ at room temperature. For double immunofluorescence, sections were incubated with a mixture of polyclonal and monoclonal primary antibodies, followed by a mixture of Cy3- and FITC- conjugated secondary antibodies or FITCconjugated IB4 (10 $\mu \mathrm{g} / \mathrm{ml}$; Sigma-Aldrich, Catalog: L2895) ${ }^{25}$. In some cases, DAPI (1:1000, Vector laboratories, Catalog: H-1200) or Nissl staining (1:200, ThermoFisher Scientific, Catalog: N21483) was used to stain cell nuclei or neurons in tissue sections. The stained sections were examined with a Nikon fluorescence microscope, and images were captured with a CCD Spot camera. For high resolution images, sections were also examined under a Zeiss 510 inverted confocal microscope. To confirm the specificity of PD-1 antibody, blocking experiments were conducted in DRG, nerve, spinal cord, and skin sections using a 
mixture of anti-PD- 1 antibody $(1: 500 \approx 2 \mu \mathrm{g} / \mathrm{ml})$ and immunizing blocking peptide (1:300 $\approx$ $0.7 \mu \mathrm{g} / \mathrm{ml}$, i.e. 10 fold of the mole concentration of the antibody, Sigma, Catalog: SBP4065), based on a protocol recommended for blocking with immunizing peptide (www.abcam.com/ technical).

To determine if there is neuronal loss in $P d 1$ deficient mice, we conducted semiquantification of different neuronal populations in DRGs of WT and $\mathrm{Pd}^{-1-}$ mice. All the series L4 DRG sections $(14 \mu \mathrm{m})$ were collected and every 5th section was used for respective immunostaining (CGRP, NF200), IB4 staining, or Nissl staining. The number of positive neurons for each staining was counted and the percentage of the labeled population was calculated based on the Nissl-stained total population in DRG sections. To quantify immunostaining in the dorsal horn, immunofluorescence intensity in spinal cord sections of WT and KO mice (3-5 spinal sections/per mouse) were included.

\section{ELISA}

Mouse PD-L1 ELISA kit was purchased from US Biological (Catalog: 027620). ELISA was performed using culture medium, serum and different normal tissues including paw skin, sciatic nerve, gastrocnemius, DRG, brain, spinal cord, lung, thymus, kidney, spleen, liver, as well as malignant skin tissue baring melanoma. Cultured cells and tissues were homogenized in a lysis buffer containing protease and phosphatase inhibitors. Serum was obtained from whole blood, collected by cardiac puncture. After 30 minutes at room temperature, the clot was removed in a refrigerated centrifuge at $2,000 \times \mathrm{g}$ for $10 \mathrm{~min}$ to collect the supernatant (serum). For each ELISA assay, $50 \mu \mathrm{g}$ proteins, $50 \mu \mathrm{l}$ of culture medium, or $50 \mu \mathrm{l}$ of serum were used. ELISA was conducted according to manufacturer's instructions. The standard curve was included in each experiment.

\section{Quantitative real-time RT-PCR}

Hindpaw skins of MCI-4W mice were collected $3 \mathrm{~h}$ after the intraplantar injection. Total RNA was extracted using Direct-zol ${ }^{\mathrm{TM}}$ RNA MiniPrep Kit (Zymo Research Corporation) and $0.5-1 \mu \mathrm{g}$ of RNA was reverse-transcribed using the iScript cDNA Synthesis ${ }^{\circledR}$ (BioRad). Specific primers including GAPDH control were designed using IDT SciTools RealTime PCR software. We performed gene-specific mRNA analyses using the MiniOpticon Real-Time PCR system (BioRad) ${ }^{35}$. Quantitative PCR amplification reactions contained the same amount of Reverse transcription (RT) product, including 7.5 $\mu \mathrm{L}$ of $2 \times$ iQSYBR-green mix (BioRad) and 100-300 $\mathrm{nM}$ of forward and reverse primers in a final volume of $15 \mu \mathrm{L}$. The primer sequences were shown in Supplementary Fig. 12b. Primer efficiency was obtained from the standard curve and integrated for calculation of the relative gene expression, which was based on real-time PCR threshold values of different transcripts and groups.

\section{Western blot}

Protein samples were prepared in the same way as for ELISA analysis, and 20-50 $\mu \mathrm{g}$ of proteins were loaded for each lane and separated by SDS-PAGE gel (4-15\%; Bio-Rad). After the transfer, the blots were incubated overnight at $4{ }^{\circ} \mathrm{C}$ with polyclonal antibody against PD-1 (1:1000, rabbit; Sigma, Catalog: PRS4065). For loading control, the blots were 
probed with GAPDH antibody (1:20000, mouse; Sigma, Catalog: G8795). These blots were further incubated with HRP-conjugated secondary antibody and developed in ECL solution (Pierce). Specific bands were evaluated by apparent molecular sizes. The intensity of the selected bands was analyzed using NIH Image $\mathbf{J}$ software. Uncut gels for the represented blots in were included in Supplementary Fig. 13.

\section{Whole-cell patch clamp recordings in dissociated mouse DRG neuron}

DRGs were aseptically removed from 4-7 week-old mice and digested with collagenase ( 0.2 $\mathrm{mg} / \mathrm{ml}$, Roche)/dispase-II ( $3 \mathrm{mg} / \mathrm{ml}$, Roche) for $120 \mathrm{~min}$. Cells were placed on glass cover slips coated with poly-D-lysine and grown in a neurobasal defined medium (10\% fetal bovine serum and $2 \% \mathrm{~B} 27$ supplement) at $37^{\circ} \mathrm{C}$ with $5 \% \mathrm{CO}_{2} / 95 \%$ air for $24 \mathrm{~h}$ before experiments. Whole-cell voltage clamp recordings were performed at room temperature to measure transient sodium currents and action potentials, respectively, with an EPC10 amplifier (HEKA) and an Axopatch-200B amplifier with a Digidata 1440A (Axon Instruments) ${ }^{25}$. The patch pipettes were pulled from borosilicate capillaries (Chase Scientific Glass Inc.). When filled with the pipette solution, the resistance of the pipettes was 4-5 M $\Omega$. The recording chamber (300 $\mu \mathrm{l}$ ) was continuously superfused (3-4 ml/min). Series resistance was compensated for $(>80 \%)$, and leak subtraction was performed. Data were low-pass-filtered at $2 \mathrm{KHz}$, sampled at $10 \mathrm{KHz}$. The pClamp10 (Axon Instruments) software was used during experiments and analysis. For sodium current recording, pipette solution contained (in $\mathrm{mM}$ ): $\mathrm{CsCl} 130, \mathrm{NaCl} 9, \mathrm{MgCl}_{2}$ 1, EGTA 10, HEPES 10, adjusted to $\mathrm{pH} 7.4$ with $\mathrm{CsOH}$. The external solution was composed of (in $\mathrm{mM}$ ): $\mathrm{NaCl} 131$, TEACl 10, $\mathrm{CsCl}$ $10, \mathrm{CaCl}_{2} 1, \mathrm{MgCl}_{2} 2, \mathrm{CdCl}_{2}$ 0.3, 4-aminopyridine 3, HEPES 10, glucose 10 adjusted to $\mathrm{pH}$ 7.4 with $\mathrm{NaOH}$. In voltage-clamp experiments, the transient sodium current $\left(\mathrm{I}_{\mathrm{Na}}\right)$ was evoked by a test pulse to $+0 \mathrm{mV}$ from the holding potential, $-70 \mathrm{mV}^{25}$. For action potential and resting membrane potential (RMP) recordings, pipette solution contained (in $\mathrm{mM}$ ): Kgluconate 126, $\mathrm{NaCl} 10, \mathrm{MgCl}_{2}$ 1, EGTA 10, NaATP 2, and $\mathrm{MgGTP} 0.1$, adjusted to $\mathrm{pH} 7.3$ with $\mathrm{KOH}$. The external solution was composed of (in $\mathrm{mM}$ ): $\mathrm{NaCl} 140, \mathrm{KCl} 5, \mathrm{CaCl}_{2} 2$, $\mathrm{MgCl}_{2}$ 1, HEPES 10, glucose 10, adjusted to $\mathrm{pH} 7.4$ with $\mathrm{NaOH}$. In current-clamp experiments, the action potentials were evoked by current injection steps. RMP was measured without a current injection.

\section{Whole-cell patch clamp recordings in whole mount DRGs of mice ex vivo}

L4-L5 DRGs were carefully removed 4 days after sham surgery or CCI surgery and placed in cold oxygenated ACSF. The connective tissue was gently removed under a microscope and the ganglia were digested with a mixture of $0.4 \mathrm{mg} / \mathrm{mL}$ trypsin (Sigma) and $1.0 \mathrm{mg} / \mathrm{ml}$ type-A collagenase (Sigma) for $30 \mathrm{~min}$ at $37^{\circ} \mathrm{C}$. The intact ganglia were then incubated in ACSF oxygenated with $95 \% \mathrm{O}_{2}$ and $5 \% \mathrm{CO}_{2}$ at $28{ }^{\circ} \mathrm{C}$ for at least $1 \mathrm{~h}$ before transferring them to the recording chamber. DRG neurons were visualized with a $40 \times$ water-immersion objective using a BX51WI microscope (Olympus). Whole-cell current and voltage recordings were acquired with an Axon700B amplifier. Patch pipettes (4-7 M $\Omega$ ) were pulled from borosilicate glass capillaries on P-97 puller. The recording chamber (300 $\mu \mathrm{l})$ was continuously superfused (3-4 ml/min). Series resistance was compensated for (> 80\%), and leak subtraction was performed. The pipette solution contained (in $\mathrm{mM}$ ): $140 \mathrm{KCl}, 2 \mathrm{MgCl}_{2}$, 10 Hepes, $2 \mathrm{Mg}$-ATP, pH 7.4. Osmolarity was adjusted to 290-300 mOsm. Data was 
acquired with a Digidata 1322A acquisition system (molecular devices) using pCLAMP 9.0 software. Signals were low-pass filtered at $5 \mathrm{kHz}$, sampled at $10 \mathrm{kHz}$ and analyzed offline.

\section{Primary cultures and patch clam recordings in human DRG neurons}

Non-diseased human DRGs were obtained from donors through NDRI with permission of exemption from Duke IRB. Postmortem L3-L5 DRGs were dissected from 5 male donors at the age of 27, 31, 43, 54, and 67 and delivered in ice-cold culture medium to the laboratory at Duke University within 24-72 hours of death. Upon the delivery, DRGs were rapidly dissected from nerve roots and minced in a calcium-free HBSS (Gibco). Human DRG cultures were prepared as previously reported ${ }^{25}$. DRGs were digested at $37^{\circ} \mathrm{C}$ in humidified $\mathrm{O}_{2}$ incubator for $120 \mathrm{~min}$ with collagenase Type II (Worthington, 285 units/mg, $12 \mathrm{mg} / \mathrm{ml}$ final concentration) and dispase II (Roche, $1 \mathrm{unit} / \mathrm{mg}, 20 \mathrm{mg} / \mathrm{ml}$ ) in PBS with $10 \mathrm{mM}$ HEPES, $\mathrm{pH}$ adjusted to 7.4 with $\mathrm{NaOH}$. DRGs were mechanically dissociated using firepolished pipettes, filtered through a $100 \mu \mathrm{M}$ nylon mesh and centrifuged $(500 \times \mathrm{g}$ for $5 \mathrm{~min})$. The pellet was resuspended, plated on $0.5 \mathrm{mg} / \mathrm{ml}$ poly-D-lysine-coated glass coverslips, and cells were grown in Neurobasal medium supplemented with 10\% FBS, 2\% B-27 supplement, $1 \% \mathrm{~N}-2$ supplement, and $1 \%$ penicillin/streptomycin. Whole-cell patch clamp recordings in small-diameter DRG neurons $(<55 \mu \mathrm{m})$ were conducted at room temperature using patch pipettes with resistances of $2-3 \mathrm{M} \Omega$. The recording chamber was continuously superfused (3-4 ml/min). The data were acquired at a rate of $10 \mathrm{kHz}$ and filtered at $3 \mathrm{kHz}$ using an EPC-10 amplifier (HEKA, Germany) and an Axopatch-200B amplifier with a Digidata 1440A (Axon Instruments). For sodium current recording, pipette solution contained (in $\mathrm{mM}$ ): $\mathrm{CsCl} 130, \mathrm{NaCl} 9, \mathrm{MgCl}_{2}$ 1, EGTA 10, HEPES 10, adjusted to $\mathrm{pH} 7.4$ with $\mathrm{CsOH}$. The external solution was composed of (in $\mathrm{mM}$ ): $\mathrm{NaCl} 131, \mathrm{TEACl} 10, \mathrm{CsCl}$ $10, \mathrm{CaCl}_{2} 1, \mathrm{MgCl}_{2} 2, \mathrm{CdCl}_{2}$ 0.3, 4-aminopyridine 3, HEPES 10, glucose 10 adjusted to $\mathrm{pH}$ 7.4 with $\mathrm{NaOH}$. In voltage-clamp experiments, the transient sodium current $\left(\mathrm{I}_{\mathrm{Na}}\right)$ was evoked by a test pulse to $0 \mathrm{mV}$ from the holding potential of $-70 \mathrm{mV}$. Pre-treatment of the SHP-1 inhibitor SSG was performed 30 min prior to whole-cell patch-clamp recordings. For action potential and RMP recordings, pipette solution contained (in $\mathrm{mM}$ ): K-gluconate 126, $\mathrm{NaCl} 10, \mathrm{MgCl}_{2}$ 1, EGTA 10, NaATP 2, and MgGTP 0.1, adjusted to $\mathrm{pH} 7.3$ with $\mathrm{KOH}$. The external solution was composed of (in $\mathrm{mM}$ ): $\mathrm{NaCl} 140, \mathrm{KCl} 5, \mathrm{CaCl}_{2} 2, \mathrm{MgCl}_{2} 1$, HEPES 10, glucose 10, adjusted to $\mathrm{pH} 7.4$ with $\mathrm{NaOH}$. In current-clamp experiments, the action potentials were evoked by a current injection ${ }^{25}$. The resting membrane potential was measured without a current injection.

\section{$\mathrm{CHO}$ cell culture, transfection and electrophysiology}

$\mathrm{CHO}$ cell line was purchased from Duke Cell Culture Facility. Cells were cultured in high glucose (4.5 g/L) Dulbecco's Modified Eagle's Medium containing 10\% (v/v) fetal bovine serum (Gibco). Transfection ( $1 \mu \mathrm{g}$ cDNA) was performed with Lipofectamine ${ }^{\mathrm{TM}} 2000$ Reagent (Invitrogen) at 70\% confluency and the transfected cells were cultured in the same growth medium for $48 \mathrm{~h}$ before electrophysiological and biochemical studies. PD1/PDCD1 cDNA construct (SC117011, NM_005018) and TREK2/KCNK10 cDNA construct (SC110477, NM_021161) were purchased from Origene Technologies. Whole-cell patch clamp recordings in transfected $\mathrm{CHO}$ cells were conducted at room temperature using patch pipettes with resistances of 5-6 M $\Omega$. The recording chamber was continuously superfused 
(3-4 $\mathrm{ml} / \mathrm{min}$ ). The data were acquired at a rate of $10 \mathrm{kHz}$ and filtered at $3 \mathrm{kHz}$ using an EPC-10 amplifier (HEKA, Germany). Pipette solution contained (in mM): K-gluconate 126, $\mathrm{NaCl} 10, \mathrm{MgCl}_{2}$ 1, EGTA 10, NaATP 2, and MgGTP 0.1, adjusted to $\mathrm{pH} 7.3$ with $\mathrm{KOH}$. The external solution was composed of (in $\mathrm{mM}$ ): $\mathrm{NaCl} 140, \mathrm{KCl} 5, \mathrm{CaCl}_{2} 2, \mathrm{MgCl}_{2} 1, \mathrm{HEPES}$ 10 , glucose 10 , adjusted to $\mathrm{pH} 7.4$ with $\mathrm{NaOH}$. In voltage-clamp recordings, TREK2induced currents were elicited by voltage-ramp from $-120 \mathrm{mV}$ to $+100 \mathrm{mV}$ every $10 \mathrm{~s}$ interval. In current-clamp experiments, the resting membrane potential was measured without any membrane potential compensation.

\section{Spinal cord slice preparation and patch clamp recordings in mice ex vivo}

The L3-L5 lumbar spinal cord segment was removed from mice under urethane anesthesia $(1.5-2.0 \mathrm{~g} / \mathrm{kg}$, i.p.) and kept in pre-oxygenated ice-cold artificial cerebrospinal fluid (aCSF) solution composed of (in $\mathrm{mM}$ ): $\mathrm{NaCl} 126, \mathrm{KCl} 3, \mathrm{MgCl}_{2} 1.3, \mathrm{CaCl}_{2} 2.5, \mathrm{NaHCO}_{3} 26$; $\mathrm{NaH}_{2} \mathrm{PO}_{4} 1.25$; glucose 11. Transverse slices (300-400 $\left.\mu \mathrm{m}\right)$ were cut on a vibrating microslicer. The slices were perfused with aCSF solution for at least $1 \mathrm{~h}$ prior to experiment. The whole cell patch-clamp recordings were made from lamina IIo neurons in voltage clamp mode $^{27}$. After establishing the whole-cell configuration, neurons were held at $-60 \mathrm{mV}$ to record spontaneous EPSCs (sEPSCs) in the presence of $10 \mu \mathrm{M}$ picrotoxin and $2 \mu \mathrm{M}$ strychnine. The miniature EPSCs (mEPSCs) were recorded in some neurons in the presence of $10 \mu \mathrm{M}$ picrotoxin, $2 \mu \mathrm{M}$ strychnine, and $0.5 \mu \mathrm{M}$ tetrodotoxin. The resistance of a typical patch pipette is 5-6 M $\Omega$. Signals were filtered at $2 \mathrm{kHz}$ and digitized at $10 \mathrm{kHz}$. The recording data were analyzed using Mini Analysis (Synaptosoft Inc.).

\section{Spontaneous discharge recordings in mouse sciatic nerve in vivo}

Adult male mice (25-32g) were anaesthetized with urethane (1.5 g/kg, i.p.) and monitored for loss of hind paw pinch reflex with additional injections of urethane $(0.2 \mathrm{~g} / \mathrm{kg})$. The animals were artificially ventilated with oxygen on a respirator. The left thigh was shaved and an incision made parallel to the femur. The muscle was parted by blunt forceps dissection to expose the sciatic nerve proximal to the trifurcation. A cuff electrode (Microprobes) was placed loosely around the full circumference of the sciatic nerve. Skin flaps were raised to enclose a pool of mineral oil that covered the exposed regions of nerve. The spontaneous discharges in the sciatic nerve were recorded with a microelectrode AC amplifier (1800, A-M systems), filtered (low cut-off $100 \mathrm{~Hz}$ and hi cut-off $20 \mathrm{kHz}$ ) and digitized at $20 \mathrm{kHz}$ (Digidata 1440A, Molecular Devices). Data were stored with a personal computer using pCLAMP 10 software and analyzed with Offline Sorter software (Plexon, Dallas, TX) and Origin pro 8.0 (Origin Lab). The spikes of sciatic nerve were characterized as previously reported ${ }^{25}$.

\section{Extracellular recording in rat spinal cord in vivo}

Rats were anesthetized with urethane (1.5 g/kg, i.p.), and the trachea was cannulated to allow artificial respiration. A laminectomy was performed at vertebrae T13-L1 to expose the lumbar enlargement of the spinal cord. An intrathecal catheter (PE-10) was made for drug injection. The vertebral column was rigidly fixed in the frame with clamps. The exposed spinal cord was covered by warm $\left(37^{\circ} \mathrm{C}\right)$ saline solution. After surgery, the animal was immobilized and artificially ventilated (Capstar-100, IITC Life Science, USA). End-tidal 
$\mathrm{CO}_{2}$ was maintained at 3.5 to $4.5 \%$ and the rectal temperature at $37-38^{\circ} \mathrm{C}$ by a feedback controlled heating blanket. The electrocardiogram was monitored, and the heart rate was maintained at $250-300 / \mathrm{min}$. As we reported previously ${ }^{38}$, single unit extracellular recordings were made at L4-5 segments, 300-700 $\mathrm{mm}$ from the surface of the spinal cord with a glass micropipette filled with $0.5 \mathrm{M}$ sodium acetate (impedance 8-10 $\mathrm{M} \Omega$ at 1000 $\mathrm{Hz}$ ). The micropipette was inserted perpendicularly to the spine into the dorsal horn from a point about mid-way between the midline and medial edge of the dorsal root entry zone. Each neuron was functionally identified as a wide dynamic range (WDR) neuron on the basis of their responses to innocuous or noxious mechanical stimulation to the receptive fields (RFs) in the plantar region of the hindpaw. WDR neurons responding to innocuous stimulation and to a greater degree, noxious stimulation of the RF were analyzed in the present study. The recorded signals were amplified with a microelectrode amplifier (1800 AM System, USA) and fed to computer via a CED 1401 interface for off-line analysis using the Spike 2 software (Cambridge Electronic Design, Cambridge, UK). For low-intensity mechanical stimulation, graded stimuli with von Frey filaments (4, 8, 15, and $26 \mathrm{~g}$ ) were applied for $15 \mathrm{~s}$ at $30 \mathrm{~s}$ intervals. High-intensity (pinch) stimulation with pinch produced by a clip (150g) was also applied for $15 \mathrm{~s}$. In pharmacological studies, only one cell was studied in each animal.

\section{Measurement of hindpaw melanoma growth in mice}

To assess tumor growth after melanoma cell implantation, paw volume was determined by water displacement plethysmometer (Ugo Basile, Italy). The Plethysmometer is a microcontrolled volume meter, specially designed for accurate measurement of the rodent paw swelling. It consists of a water filled Perspex cell into which the paw is dipped. A transducer of original design records small differences in water level, caused by volume displacement. The digital read-out shows the exact volume of the paw.

\section{Behavioral analysis in mice and rats}

The following behavioral measurements were conducted in a blinded manner and during daytime (light cycle) normally starting at 9 AM.

Spontaneous pain in mouse melanoma model-We measured the time (seconds) mice spent on licking or flinching the melanoma-bearing hindpaws for 1 or 3 hours.

Von Frey test for mechanical pain-Animals were habituated to the testing environment daily for at least 2 days before baseline testing. The room temperature and humidity remained stable for all experiments. For testing mechanical sensitivity, we confined mice in boxes placed on an elevated metal mesh floor and stimulated their hindpaws with a series of von Frey hairs with logarithmically increasing stiffness (0.02$2.56 \mathrm{~g}$, Stoelting), presented perpendicularly to the central plantar surface. We determined the $50 \%$ paw withdrawal threshold by up-down method $^{35}$.

Hargreaves test for thermal pain-Thermal sensitivity was tested using Hargreaves radiant heat apparatus (IITC Life Science), the basal paw withdrawal latency was adjusted to 9-12 s, with a cutoff of $20 \mathrm{~s}$ to prevent tissue damage ${ }^{35}$. 
Rota-rod test for motor function-A Rota-rod system (IITC Life Science Inc.) was used to assess the motor function. Mice were tested for three trails separated by $10 \mathrm{~min}$ intervals. During the tests, the speed of rotation was accelerated from 2 to 20 r.p.m. in 3 min. The falling latency was recorded and averaged ${ }^{35}$.

\section{Conditioned place preference (CPP) test for spontaneous/ongoing pain-We}

used a single trial conditioning protocol to measure $\mathrm{CPP}^{35}$. All mice underwent a 3-day preconditioning habituation and animal behavior was video-recorded. Analyses of the preconditioning (baseline) behavior showed no pre-existing chamber preference. On the conditioning day, mice received the vehicle (PBS, $20 \mu \mathrm{l}$, i.pl.) paired with a randomly chosen chamber in the morning, and PD-1 (5 $\mu$ in $20 \mu \mathrm{l}$ PBS, i.pl.) paired with the other chamber 4 $\mathrm{h}$ later. Chamber pairings were counterbalanced. On the test day, $20 \mathrm{~h}$ following the afternoon pairing, mice were placed in the CPP test box with access to both chambers and the behavior was recorded for $15 \mathrm{~min}$ and analyzed by ANY-maze software for chamber preference.

\section{Statistical analyses}

All the data were expressed as mean \pm s.e.m, as indicated in the figure legends. The sample size for each experiment was based on our previous studies on such experiment ${ }^{25,35}$. Statistical analyses were completed with Prism GraphPad 5.0. Biochemical and behavioral data were analyzed using two-tailed student's t-test (two groups) or Two-Way ANOVA followed by post-hoc Bonferroni test. Electrophysiological data were tested using one-way ANOVA (for multiple comparisons) or Two-Way ANOVA (for multiple time points) followed by post-hoc Bonferroni test or student's t-test (two groups). The criterion for statistical significance was $P<0.05$.

The data that support the findings of this study are available from the corresponding author upon reasonable request.

\section{Supplementary Material}

Refer to Web version on PubMed Central for supplementary material.

\section{Acknowledgments}

This study is supported by NIH RO1 grants NS87988, DE17794, and DE22743 and National Science Fund of China (NSFC) 31420103903. Y.H.K. was supported by the National Research Foundation of Korea (NRF) 2013R1A6A3A04065858.

\section{Reference List}

1. Mantyh PW. Cancer pain and its impact on diagnosis, survival and quality of life. Nat. Rev. Neurosci. 2006; 7:797-809. [PubMed: 16988655]

2. Mantyh P. Bone cancer pain: causes, consequences, and therapeutic opportunities. Pain. 2013; 154(Suppl 1):S54-S62. [PubMed: 23916671]

3. Selvaraj D, et al. A Functional Role for VEGFR1 Expressed in Peripheral Sensory Neurons in Cancer Pain. Cancer Cell. 2015; 27:780-796. [PubMed: 26058077] 
4. Jimenez-Andrade JM, Ghilardi JR, Castaneda-Corral G, Kuskowski MA, Mantyh PW. Preventive or late administration of anti-NGF therapy attenuates tumor-induced nerve sprouting, neuroma formation, and cancer pain. Pain. 2011; 152:2564-2574. [PubMed: 21907491]

5. Cain DM, et al. Functional interactions between tumor and peripheral nerve: changes in excitability and morphology of primary afferent fibers in a murine model of cancer pain. J Neurosci. 2001; 21:9367-9376. [PubMed: 11717370]

6. Schweizerhof M, et al. Hematopoietic colony-stimulating factors mediate tumor-nerve interactions and bone cancer pain. Nat. Med. 2009; 15:802-807. [PubMed: 19525966]

7. Schmidt BL. The neurobiology of cancer pain. Neuroscientist. 2014; 20:546-562. [PubMed: 24664352]

8. Brahmer JR, et al. Safety and activity of anti-PD-L1 antibody in patients with advanced cancer. N. Engl. J Med. 2012; 366:2455-2465. [PubMed: 22658128]

9. Negin BP, et al. Symptoms and signs of primary melanoma: important indicators of Breslow depth. Cancer. 2003; 98:344-348. [PubMed: 12872355]

10. Ji RR, Chamessian A, Zhang YQ. Pain regulation by non-neuronal cells and inflammation. Science. 2016; 354:572-577. [PubMed: 27811267]

11. Sharma P, Allison JP. The future of immune checkpoint therapy. Science. 2015; 348:56-61. [PubMed: 25838373]

12. Butte MJ, Keir ME, Phamduy TB, Sharpe AH, Freeman GJ. Programmed death-1 ligand 1 interacts specifically with the B7-1 costimulatory molecule to inhibit T cell responses. Immunity. 2007; 27:111-122. [PubMed: 17629517]

13. Keir ME, Butte MJ, Freeman GJ, Sharpe AH. PD-1 and its ligands in tolerance and immunity. Annu. Rev. Immunol. 2008; 26:677-704. [PubMed: 18173375]

14. Day CL, et al. PD-1 expression on HIV-specific T cells is associated with T-cell exhaustion and disease progression. Nature. 2006; 443:350-354. [PubMed: 16921384]

15. Herbs RS, et al. Predictive correlates of response to the anti-PD-L1 antibody MPDL3280A in cancer patients. Nature. 2014; 515:563-567. [PubMed: 25428504]

16. Topalian SL, et al. Safety, activity, and immune correlates of anti-PD-1 antibody in cancer. N. Engl. J Med. 2012; 366:2443-2454. [PubMed: 22658127]

17. Ansell SM, et al. PD-1 blockade with nivolumab in relapsed or refractory Hodgkin's lymphoma. N. Engl. J Med. 2015; 372:311-319. [PubMed: 25482239]

18. Hamanishi J, et al. Safety and Antitumor Activity of Anti-PD-1 Antibody, Nivolumab, in Patients With Platinum-Resistant Ovarian Cancer. J Clin. Oncol. 2015

19. Postow MA, et al. Nivolumab and ipilimumab versus ipilimumab in untreated melanoma. N. Engl. J. Med. 2015; 372:2006-2017. [PubMed: 25891304]

20. Talbot S, Foster SL, Woolf CJ. Neuroimmunity: Physiology and Pathology. Annual review of immunology. 2016; 34:421-447.

21. McMahon SB, La Russa F, Bennett DL. Crosstalk between the nociceptive and immune systems in host defence and disease. Nature reviews. Neuroscience. 2015; 16:389-402. [PubMed: 26087680]

22. Chiu IM, et al. Bacteria activate sensory neurons that modulate pain and inflammation. Nature. 2013; 501:52-57. [PubMed: 23965627]

23. Ji RR, Xu ZZ, Gao YJ. Emerging targets in neuroinflammation-driven chronic pain. Nat. Rev. Drug Discov. 2014; 13:533-548. [PubMed: 24948120]

24. Li Y, et al. Toll-like receptor 4 signaling contributes to Paclitaxel-induced peripheral neuropathy. J Pain. 2014; 15:712-725. [PubMed: 24755282]

25. Xu ZZ, et al. Inhibition of mechanical allodynia in neuropathic pain by TLR5-mediated A-fiber blockade. Nat. Med. 2015

26. Park CK, et al. Extracellular MicroRNAs Activate Nociceptor Neurons to Elicit Pain via TLR7 and TRPA1. Neuron. 2014; 82:47-54. [PubMed: 24698267]

27. Berta T, et al. Extracellular caspase- 6 drives murine inflammatory pain via microglial TNF-alpha secretion. J Clin. Invest. 2014; 124:1173-1186. [PubMed: 24531553]

28. Patel SP, Kurzrock R. PD-L1 Expression as a Predictive Biomarker in Cancer Immunotherapy. Molecular cancer therapeutics. 2015; 14:847-856. [PubMed: 25695955] 
29. Weber JS, et al. Nivolumab versus chemotherapy in patients with advanced melanoma who progressed after anti-CTLA-4 treatment (CheckMate 037): a randomised, controlled, open-label, phase 3 trial. Lancet Oncol. 2015; 16:375-384. [PubMed: 25795410]

30. Brahmer JR, Hammers H, Lipson EJ. Nivolumab: targeting PD-1 to bolster antitumor immunity. Future.Oncol. 2015; 11:1307-1326. [PubMed: 25798726]

31. Hucho T, Levine JD. Signaling pathways in sensitization: toward a nociceptor cell biology. Neuron. 2007; 55:365-376. [PubMed: 17678851]

32. Reichling DB, Levine JD. Critical role of nociceptor plasticity in chronic pain. Trends Neurosci. 2009; 32:611-618. [PubMed: 19781793]

33. Basbaum AI, Bautista DM, Scherrer G, Julius D. Cellular and molecular mechanisms of pain. Cell. 2009; 139:267-284. [PubMed: 19837031]

34. Devor M, Wall PD, Catalan N. Systemic lidocaine silences ectopic neuroma and DRG discharge without blocking nerve conduction. Pain. 1992; 48:261-268. [PubMed: 1589245]

35. Chen G, Park CK, Xie RG, Ji RR. Intrathecal bone marrow stromal cells inhibit neuropathic pain via TGF-beta secretion. J Clin. Invest. 2015; 125:3226-3240. [PubMed: 26168219]

36. Todd AJ. Neuronal circuitry for pain processing in the dorsal horn. Nat. Rev. Neurosci. 2010; 11:823-836. [PubMed: 21068766]

37. Braz J, Solorzano C, Wang X, Basbaum AI. Transmitting Pain and Itch Messages: A Contemporary View of the Spinal Cord Circuits that Generate Gate Control. Neuron. 2014; 82:522-536. [PubMed: 24811377]

38. Yang Y, et al. Delayed Activation of Spinal Microglia Contributes to the Maintenance of Bone Cancer Pain in Female Wistar Rats via P2X7 Receptor and IL-18. J. Neurosci. 2015; 35:7950 7963. [PubMed: 25995479]

39. Hebeisen M, et al. SHP-1 phosphatase activity counteracts increased T cell receptor affinity. J Clin. Invest. 2013; 123:1044-1056. [PubMed: 23391724]

40. Bennett DL, Woods CG. Painful and painless channelopathies. The Lancet. Neurology. 2014; 13:587-599. [PubMed: 24813307]

41. Acosta C, et al. TREK2 expressed selectively in IB4-binding C-fiber nociceptors hyperpolarizes their membrane potentials and limits spontaneous pain. J Neurosci. 2014; 34:1494-1509. [PubMed: 24453337]

42. Woolf CJ. Overcoming obstacles to developing new analgesics. Nat. Med. 2010; 16:1241-1247. [PubMed: 20948534]

43. Mogil JS. Animal models of pain: progress and challenges. Nat. Rev. Neurosci. 2009; 10:283-294. [PubMed: 19259101]

44. Han Q, et al. SHANK3 Deficiency Impairs Heat Hyperalgesia and TRPV1 Signaling in Primary Sensory Neurons. Neuron. 2016

45. Kleffel S, et al. Melanoma Cell-Intrinsic PD-1 Receptor Functions Promote Tumor Growth. Cell. 2015; 162:1242-1256. [PubMed: 26359984]

46. Scholz J, Woolf CJ. The neuropathic pain triad: neurons, immune cells and glia. Nat. Neurosci. 2007; 10:1361-1368. [PubMed: 17965656]

47. Uceyler N, et al. Deficiency of the negative immune regulator B7-H1 enhances inflammation and neuropathic pain after chronic constriction injury of mouse sciatic nerve. Exp. Neurol. 2010; 222:153-160. [PubMed: 20051242]

48. Guan Z, et al. Injured sensory neuron-derived CSF1 induces microglial proliferation and DAP12dependent pain. Nature neuroscience. 2016; 19:94-101. [PubMed: 26642091]

49. Grace PM, Hutchinson MR, Maier SF, Watkins LR. Pathological pain and the neuroimmune interface. Nat. Rev. Immunol. 2014

50. Sorge RE, et al. Different immune cells mediate mechanical pain hypersensitivity in male and female mice. Nat. Neurosci. 2015; 18:1081-1083. [PubMed: 26120961] 
a

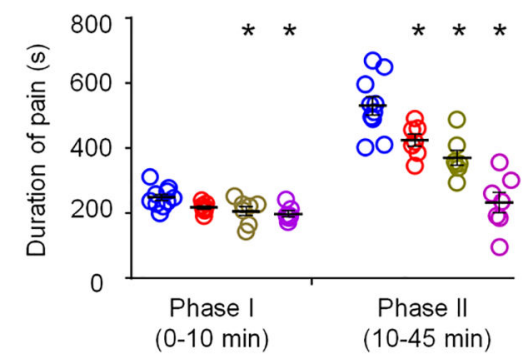
threshold in naïve mice b

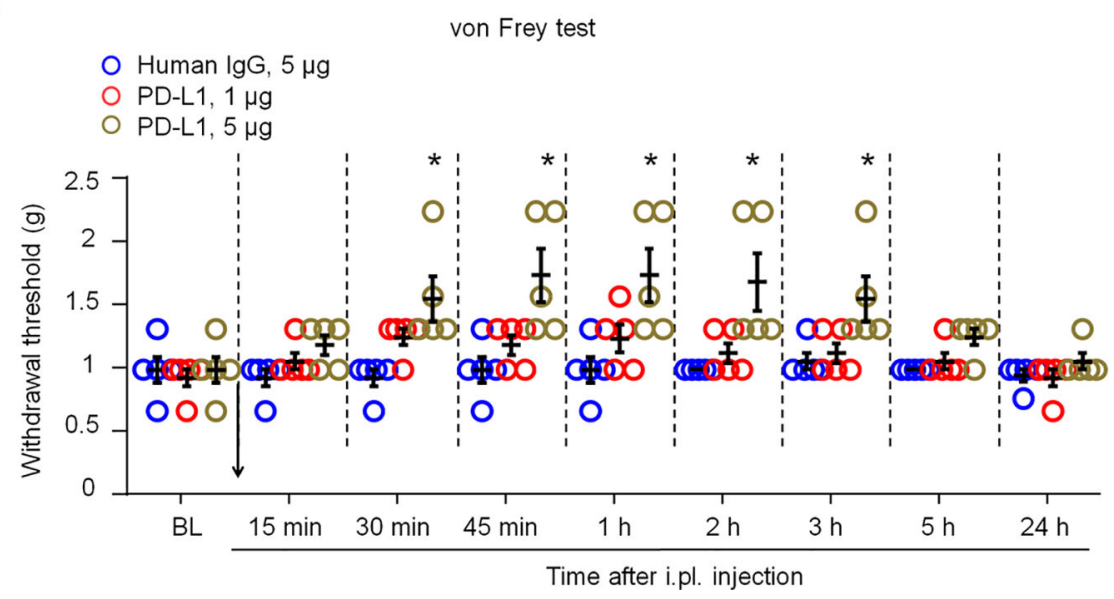

Figure 1. Exogenous PD-L1 inhibits formalin-induced inflammatory pain and increases pain

(a) Formalin-induced Phase-I and Phase-II inflammatory pain, as measured by duration of spontaneous pain behavior (flinching/licking) in every $5 \mathrm{~min}$, is reduced by intraplantar (i.pl.) pretreatment of PD-L1 (1-10 $\mu$ g). ${ }^{*} P<0.05$, vs. vehicle (PBS), One-Way ANOVA, $\mathrm{n}=$ 7-10 mice/group. PD-L1 was administered 30 min prior to the formalin injection. (b) Basal mechanical pain assessed in von Frey test in naive mice. Notice an increase in paw withdrawal threshold after PD-L1 injection (1 and $5 \mu \mathrm{g}$, i.pl.). $* P<0.05$, vs. human IgG, repeated measures Two-Way ANOVA, $\mathrm{n}=5$ mice/group. Arrow indicates drug injection. Data are mean \pm s.e.m. 
a

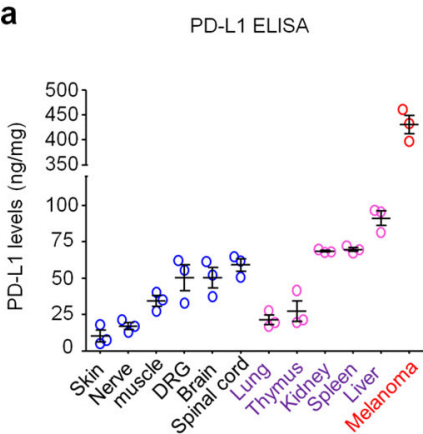

b

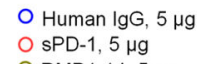

O Human IgG, $5 \mu \mathrm{gg}$ O SPD-1, $5 \mu \mathrm{g}$

O RMP1-14, $5 \mu \mathrm{g}$

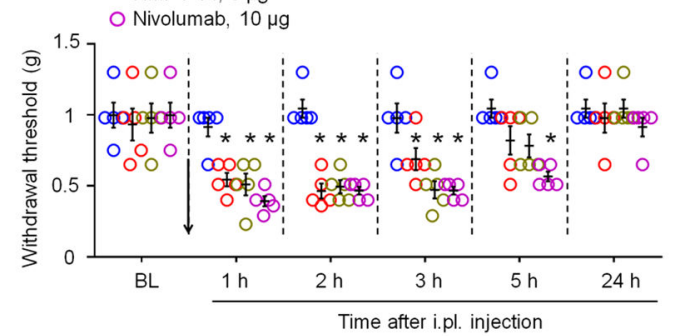

C
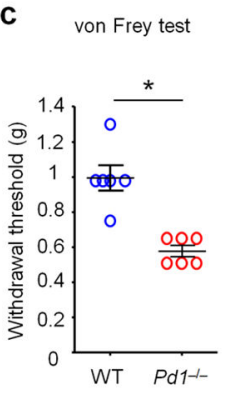

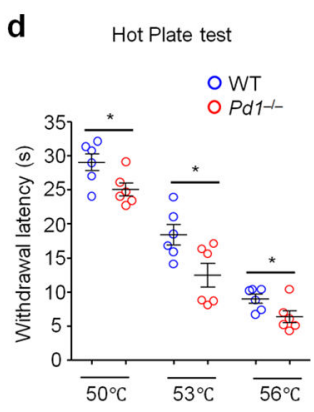

Figure 2. Endogenous PD-L1 regulates pain sensitivity in naive mice via PD-1

(a) ELISA analysis showing endogenous expression of PD-L1 in non-malignant tissues of naïve mice and melanoma tissue removed from a mouse hindpaw $4 \mathrm{w}$ after melanoma cell inoculation. Note that PD-L1 is widely expressed in various non-malignant tissues. $\mathrm{n}=3$ mice/group. (b) Inhibition of endogenous PD-L1 and PD-1 induces mechanical allodynia in naïve mice. PD-L1 was neutralized with soluble PD-1 (sPD-1, $5 \mu \mathrm{g}$, i.pl.), and PD-1 was blocked by monoclonal antibodies RMP1-14 (mouse anti-PD-1 antibody, $5 \mu \mathrm{g}$, i.pl.) and Nivolumab (human anti-PD-1 antibody, $10 \mu$ g, i.pl.). $* P<0.05$, vs. human IgG, repeated measures Two-Way ANOVA, $\mathrm{n}=5$ mice/group. Arrow indicates drug injection. (c,d) Reduced mechanical and thermal pain threshold in $\mathrm{PdI}^{-1-}$ mice, as shown in von Frey test (c) and hot plate test (d). $* P<0.05$, Two-tailed student $\mathrm{t}$-test, $\mathrm{n}=6$ mice/group. Data are mean \pm s.e.m. 

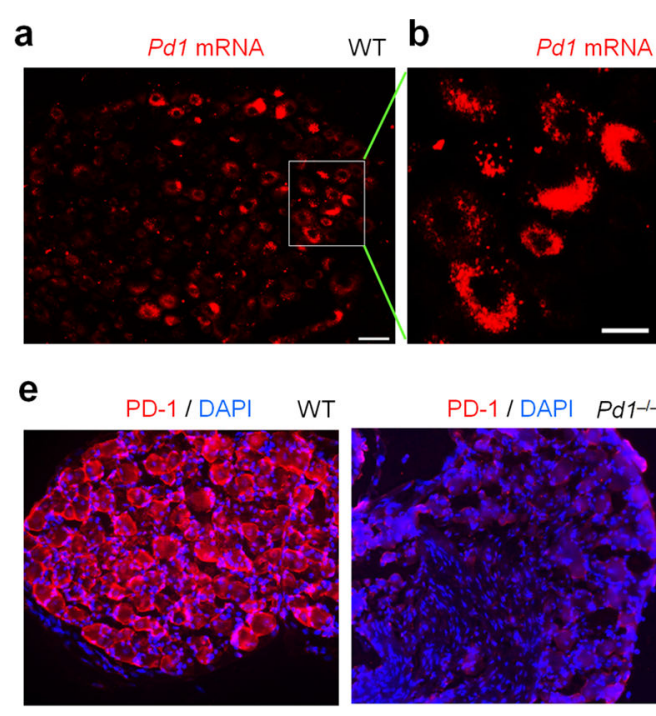

g
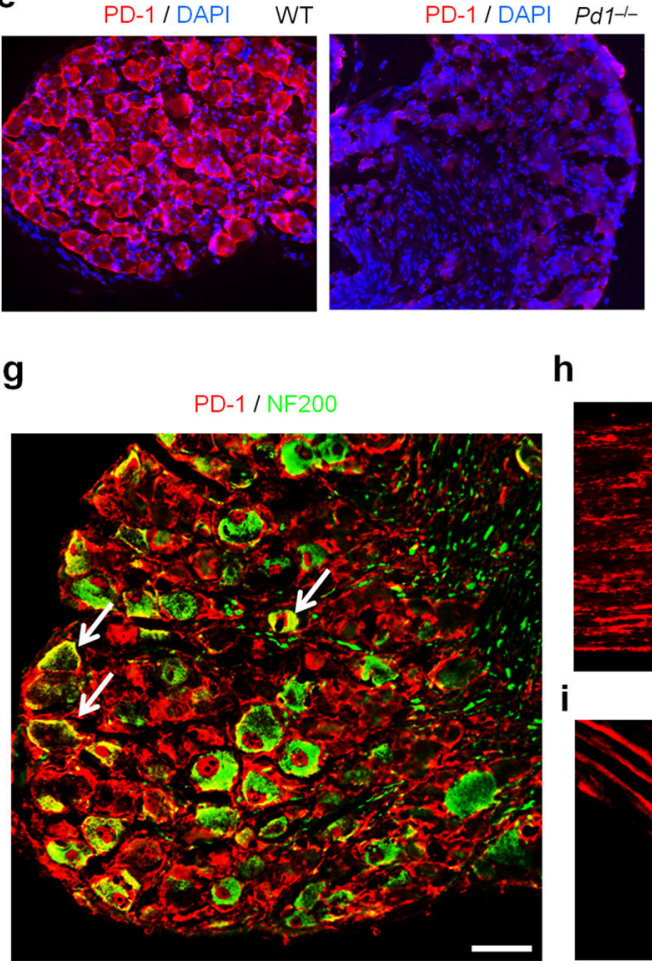

h

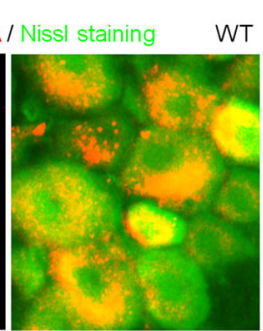

c

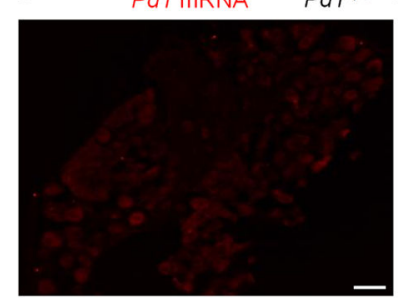

f d

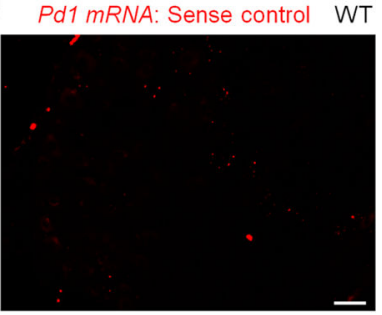

Q Total neurons

I $\mathrm{PD}-1^{+}$neurons
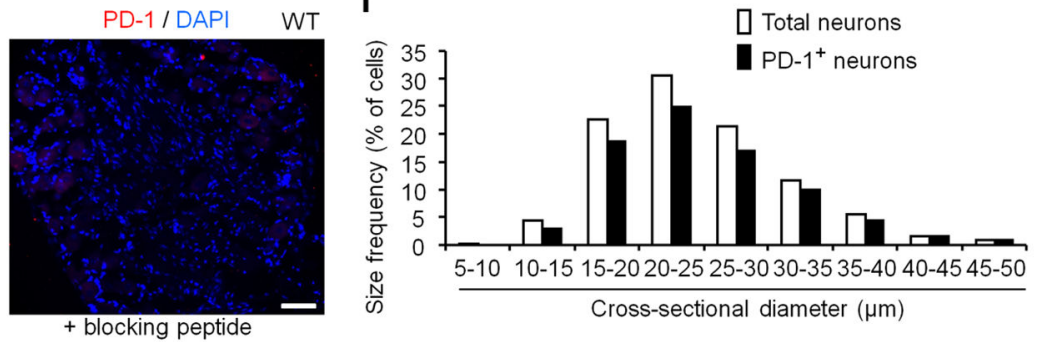

PD-1

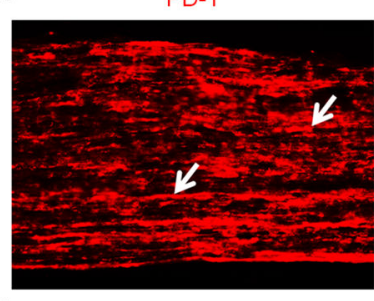

PD-1

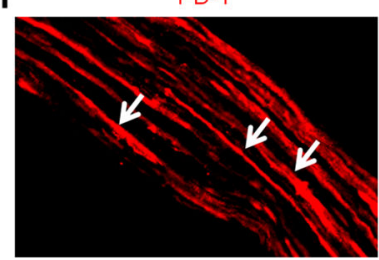

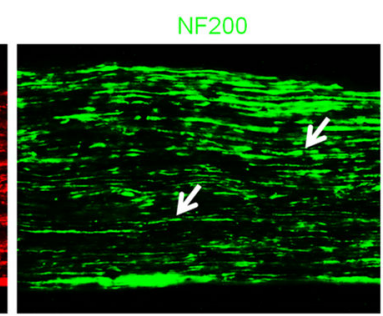

CGRP

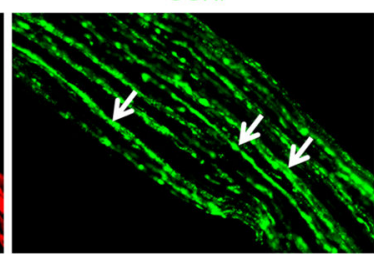

PD-1 / NF200

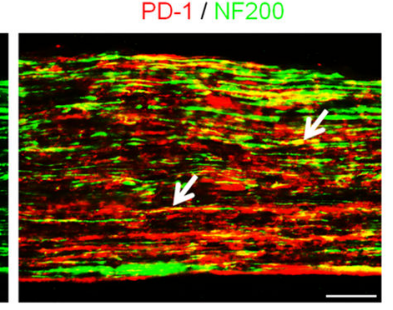

PD-1 / CGRP

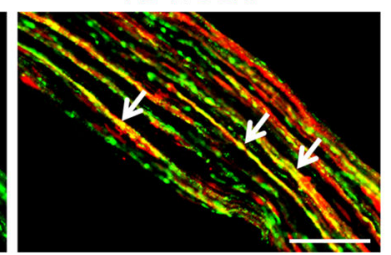

Figure 3. PD-1 is expressed by mouse DRG neurons and nerve axons

(a-d) In situ hybridization (ISH) images showing Pd1 mRNA expression in DRG of wildtype (WT) not $P d 1$ knockout $\left(P d 1^{--}\right)$mice. (a) Low magnification image of ISH with antisense probe showing Pd1 mRNA in DRG neurons of WT mice. Scale, $50 \mu \mathrm{m}$. (b) High magnification image of double ISH (red) and Nissl staining (green) in DRG sections. Scale, $20 \mu \mathrm{m}$. (c) ISH image showing loss of $P d 1 \mathrm{mRNA}$ expression in DRG neurons in $P d 1^{-1-}$ mice. Scale, $50 \mu \mathrm{m}$. (d) ISH image of sense control probe. Scale, $50 \mu \mathrm{m}$. (e) Left, image of immunostaining showing broad PD-1 expression in mouse DRG neurons. Middle, PD-1 expression is lost in $\mathrm{PdI}^{-/-}$mice. Right, absence of PD-1 immunostaining by the treatment of a blocking peptide. Blue DAPI staining shows all the cell nuclei in DRG sections. Scale, $50 \mu \mathrm{m}$. (f) Size frequency distribution of PD-1-positive and total neurons in mouse DRGs. A total of 1555 neurons from 4 WT mice were analyzed. (g,h) Double staining of PD-1 and NF200 in DRG (g) and sciatic nerve (h) sections of mice. Note that PD-1 expression in both NF200-positive and NF200-negative DRG neurons and sciatic nerve axons. Scales, $50 \mu \mathrm{m}$. (i) Double immunostaining of PD-1 and CGRP in mouse sciatic nerve. PD-1 is present in axons co-expressing CGRP. Scale, $50 \mu \mathrm{m}$. Arrows in $\mathrm{g}-\mathrm{i}$ indicate the double-labeled neurons and axons. 

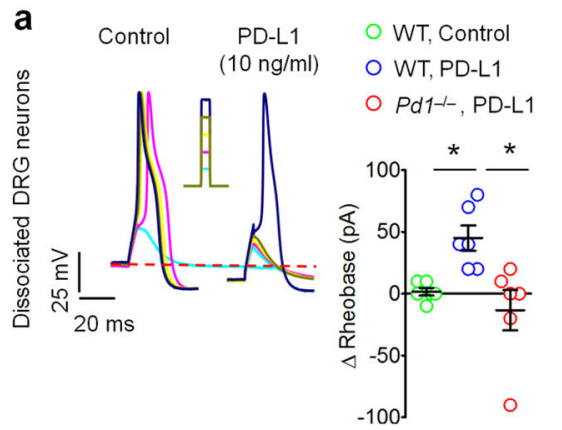

b
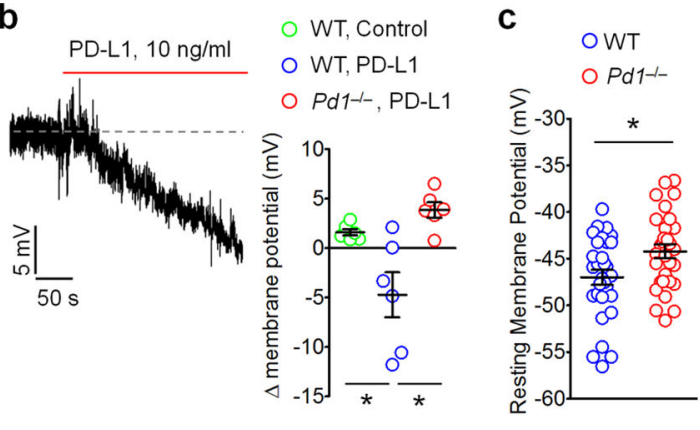

d OWT $\mathrm{Pd1--}$ * *
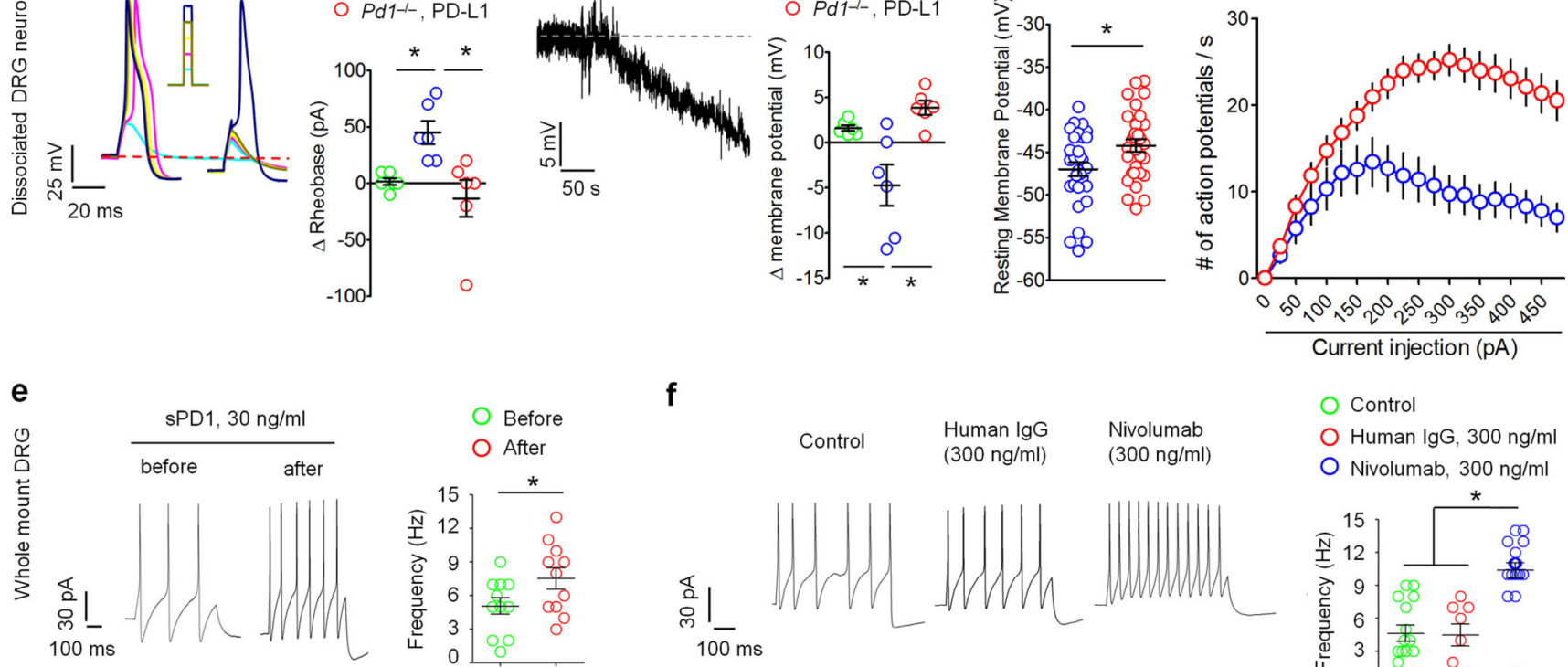

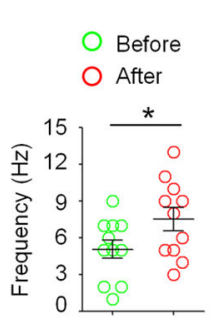

$\mathbf{f}$

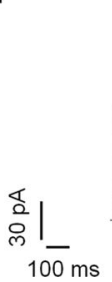

Control $(300 \mathrm{ng} / \mathrm{ml})$

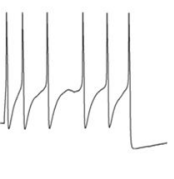

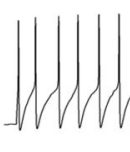

O Control

O Human IgG, $300 \mathrm{ng} / \mathrm{ml}$

O Nivolumab, $300 \mathrm{ng} / \mathrm{ml}$

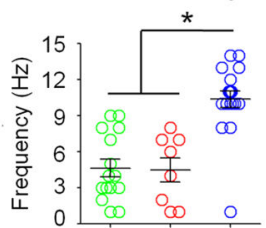

Figure 4. PD-L1 suppresses neuronal excitability in mouse DRG neurons via PD-1

(a-f) Patch clamp recordings in dissociated (a-d) and whole-mount (e,f) mouse DRG neurons with small diameters $(<25 \mu \mathrm{m})$. (a) Left, traces of action potentials (AP) showing an inhibitory effect of PD-L1 $(10 \mathrm{ng} / \mathrm{ml})$ in WT neurons. Current injection for AP induction starts from $+10 \mathrm{pA}$ and increases $10 \mathrm{pA}$ per step. Right, rheobase change in WT and Pd1-1mice. $\mathrm{n}=6$ neurons/2 mice. (b) PD-L1 induces hyperpolarization of the resting membrane potential (RMP). Right, change of RMP in WT and $P d 1^{-1-}$ mice. $\mathrm{n}=6$ neurons $/ 2$ mice. Note that PD-L1 fails to suppress action potential (a) and alter RMP (b) in $\mathrm{Pd1^{-1 }}$ mice. (c,d) Altered RMP and increased excitability in DRG neurons of $P d 1^{-1-}$ mice. (c) RMP in WT and $P d 1^{-1-}$ mice. ${ }^{*} P<0.05$, paired two-tailed t-test, $\mathrm{n}=30$ neurons $/ 2$ mice. (d) Number of action potentials evoked by current injection in WT and $P d 1^{-1-}$ mice. ${ }^{*} P<0.05$, Two-Way ANOVA followed Bonferroni's post-hoc test, $\mathrm{n}=30$ neurons/2 mice. (e) Whole-mount DRG recording showing increased action potential firing in small-sized DRG neurons after perfusion of sPD-1 (30 ng/ml). Left, traces of evoked action potential before and after sPD-1 perfusion. Right, action potential frequency following sPD-1 perfusion. $* P<0.05$, paired two-tailed Student's t-test, $\mathrm{n}=11$ neurons/3 mice. (f) Whole-mount DRG recording showing increased action potential firing in small-sized neurons following Nivolumab incubation (2 $\mathrm{h}, 300 \mathrm{ng} / \mathrm{ml}$ ). Left, traces of evoked action potential in neurons incubated with control (artificial CSF), human IgG and Nivolumab. Right, frequency of action potentials showing the effects of human IgG and Nivolumab. $* P<0.05$, vs. control and human IgG, One-Way ANOVA, followed by Bonferroni's post-hoc test, $\mathrm{n}=8-18$ neurons/3 mice. Data are mean \pm s.e.m. 
a

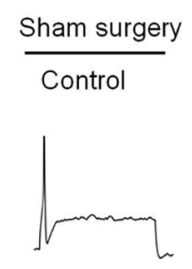

C
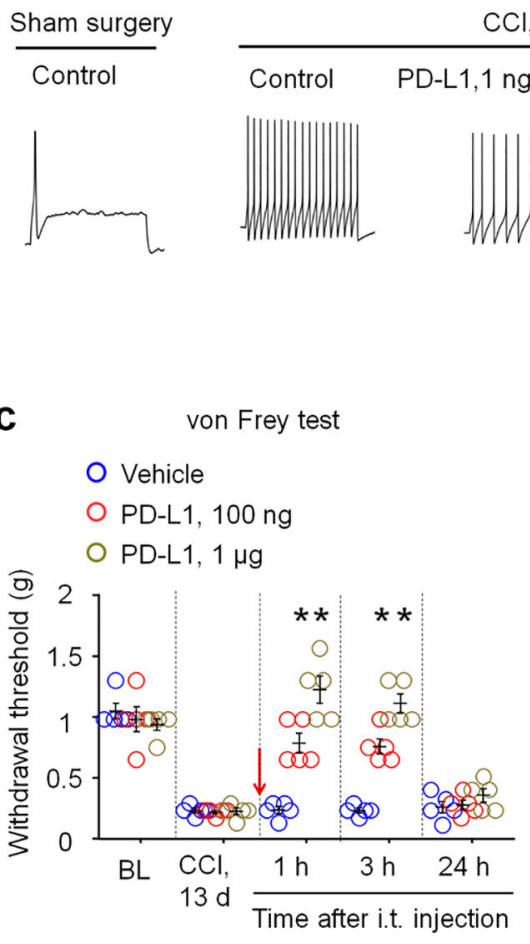

Mouse DRG neurons
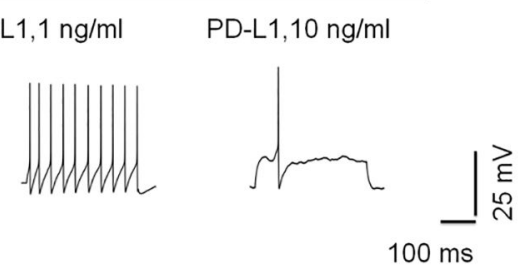

d

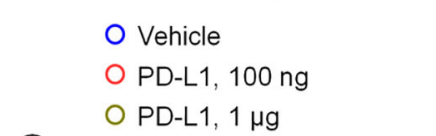

$\widehat{\infty}$

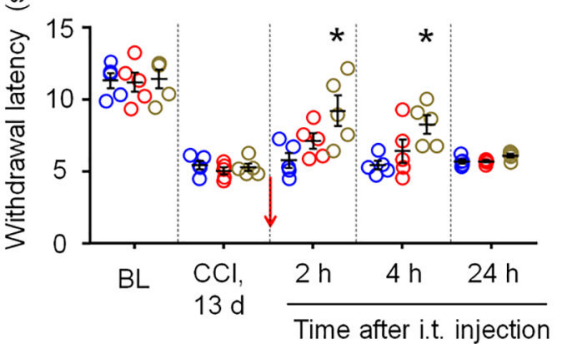

b

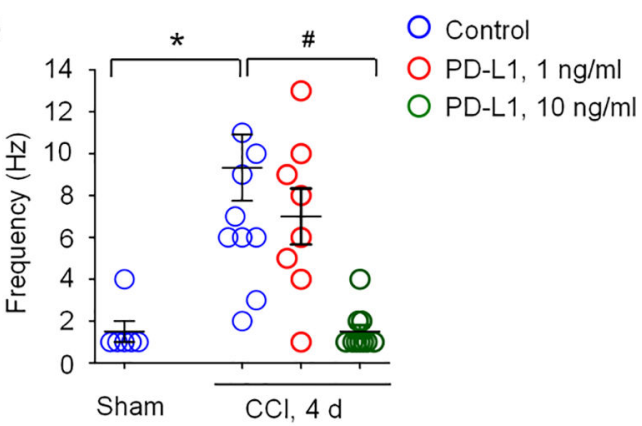

Figure 5. PD-L1 inhibits neuronal hyperexcitability and neuropathic pain after nerve injury $(\mathbf{a}, \mathbf{b})$ PD-L1 blocks the CCI-induced increases in action potential frequency in smalldiameter neurons of whole-mount DRG. (a) Traces of action potentials $4 \mathrm{~d}$ after chronic constriction injury (CCI) and the effects of PD-L1 (1 and $10 \mathrm{ng} / \mathrm{ml})$. (b) Frequency of action potentials. ${ }^{*} P<0.05$, vs. sham control, ${ }^{\#} P<0.05$, vs. control (no treatment), One-Way ANOVA, $\mathrm{n}=6-9$ neurons/group. (c,d) Intrathecal PD-L1 inhibits CCI-induced mechanical allodynia (c) and thermal hyperalgesia (d). $* P<0.05$, vs. vehicle, repeated measures TwoWay ANOVA, $\mathrm{n}=5$ mice/group. Arrow indicates drug injection. (e) Randall-Selitto test showing increased baseline mechanical pain threshold after intrathecal PD-L1 injection in naïve mice. ${ }^{*} P<0.05$, vs. vehicle, ${ }^{\#} P<0.05$, vs. baseline (BL), repeated measures Two-Way ANOVA, $\mathrm{n}=5$ mice/group. Arrow indicates drug injection. Data are mean \pm s.e.m. 
a

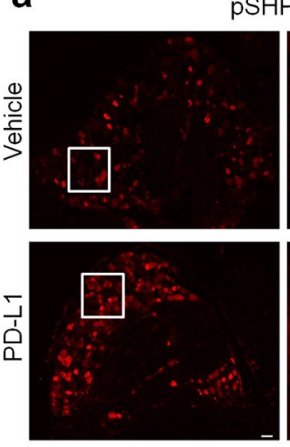

pSHP-1

C

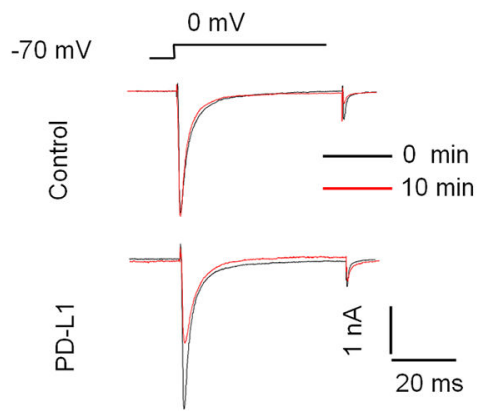

d
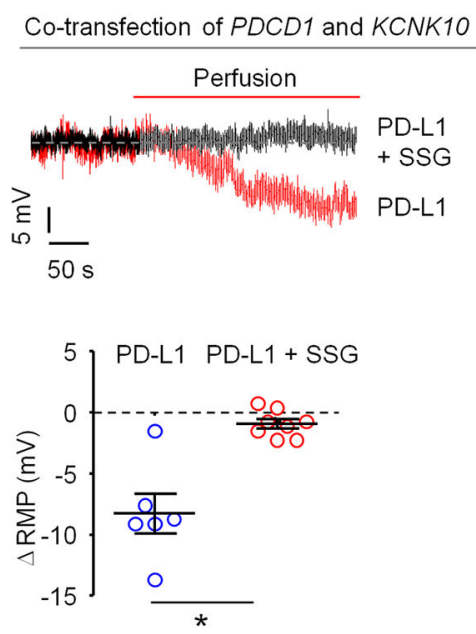
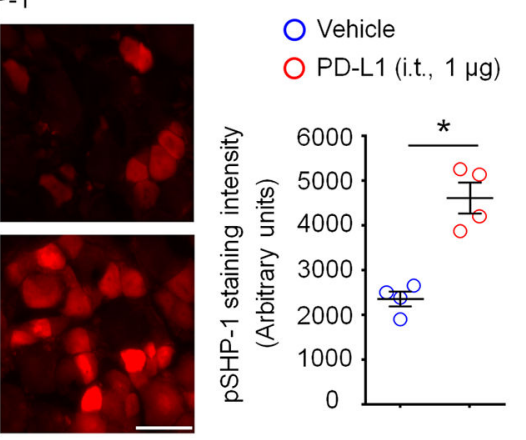

b

$$
\begin{aligned}
& O \text { Vehicle } \\
& \text { O PD-L1, } 5 \mu \mathrm{g} \\
& \bigcirc \text { PD-L1 }(5 \mu \mathrm{g})+\text { SSG }(0.5 \mu \mathrm{g}) \\
& \bigcirc \mathrm{SSG}, 0.5 \mu \mathrm{g}
\end{aligned}
$$

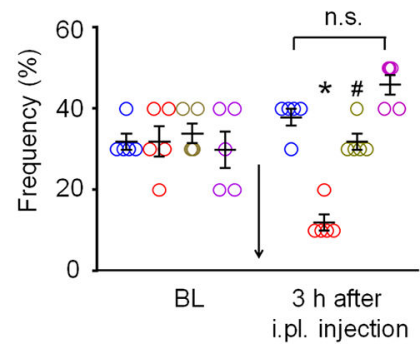

e
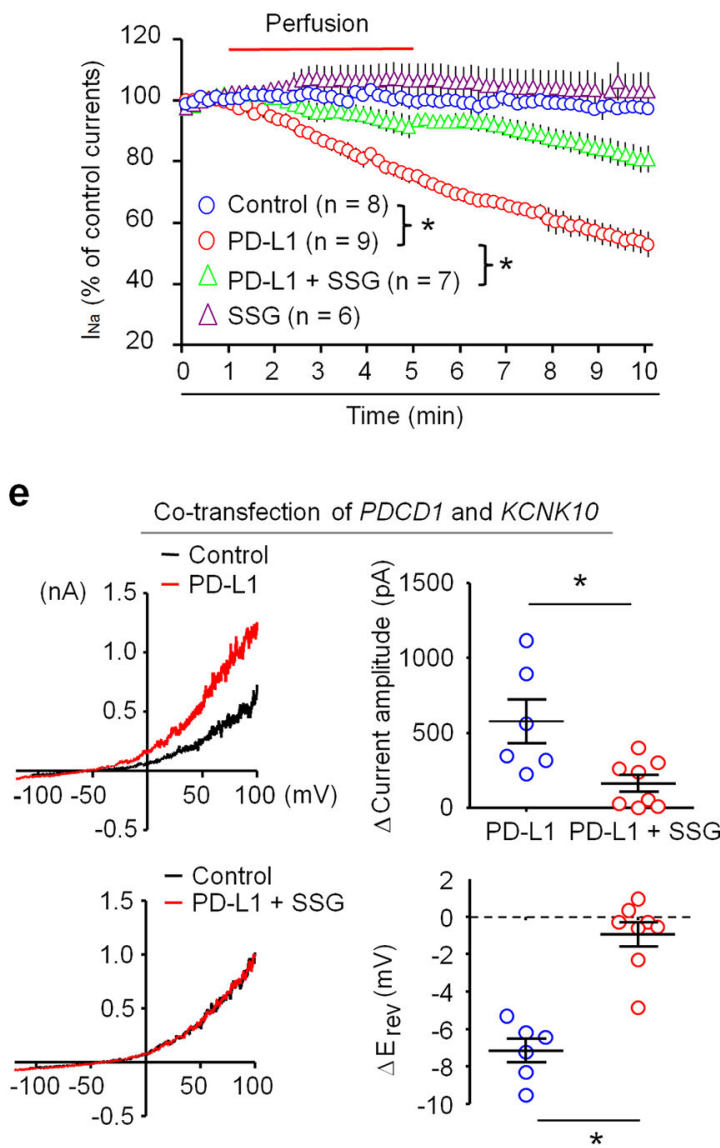

Figure 6. PD-L1 modulates neuronal excitability and pain via SHP-1

(a) Intrathecal PD-L1 (i.t. $1 \mu \mathrm{g}, 30 \mathrm{~min}$ ) increased phosphorylation of SHP-1 (pSHP-1) in mouse DRG neurons. Left, images of pSHP-1 immunostaining in vehicle and PD-L1 treated group. Scale, $50 \mu \mathrm{m}$. Middle, enlarged images from the boxes. Scale, $50 \mu \mathrm{m}$. Right, intensity of immunofluorescence of pSHP- $1^{+}$neurons. ${ }^{*} P<0.05$, Two-tailed t-test, $\mathrm{n}=4$ mice/group. (b) Paw withdrawal frequency to a $0.6 \mathrm{~g}$ filament in naïve mice and the effects of i.pl. SSG (SHP-1 inhibitor), PD-L1, and PD-L1 plus SSG in naïve mice. Note that PD-L1 induced analgesia is abolished by SSG. ${ }^{*} P<0.05$, vs. vehicle (PBS), ${ }^{\#} P<0.05$, vs. PD-L1, n.s., no significance, One-Way ANOVA, $\mathrm{n}=5$ mice/group. (c) Inhibition of transient sodium 
currents by PD-L1 $(10 \mathrm{ng} / \mathrm{ml})$ in dissociated DRG neurons and the effect of SSG $(11 \mu \mathrm{M})$. Left, traces of sodium currents. Right, time course of relative sodium currents. $* P<0.05$, Two-Way repeated measures ANOVA, $\mathrm{n}=6-9$ neurons/2 mice. (d) Regulation of RMP by PD-L1 $(10 \mathrm{ng} / \mathrm{ml})$ and its blockade SSG $(11 \mu \mathrm{M})$ in dissociated DRG neurons. $* P<0.05$, two-tailed Student's t-test, $\mathrm{n}=6-8$ neurons/2 mice. (e) PD-L1 increases TREK2 activity via SHP-1 in CHO cells. Left, traces of TREK2-induced outward currents and the effects of PDL1 and SSG. Right, quantification of outward currents and RMP changes. $* P<0.05$, twotailed Student's t-test, $n=6-8$ cells $/ 2$ cultures. Data are mean \pm s.e.m. 

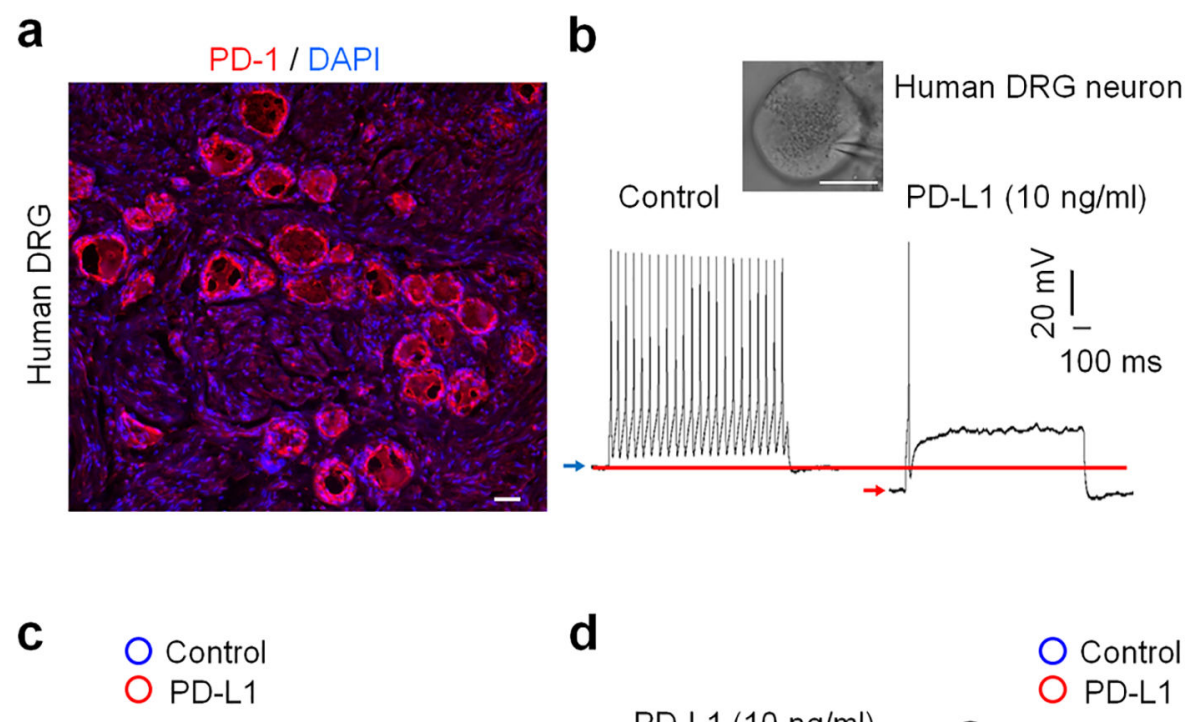

d
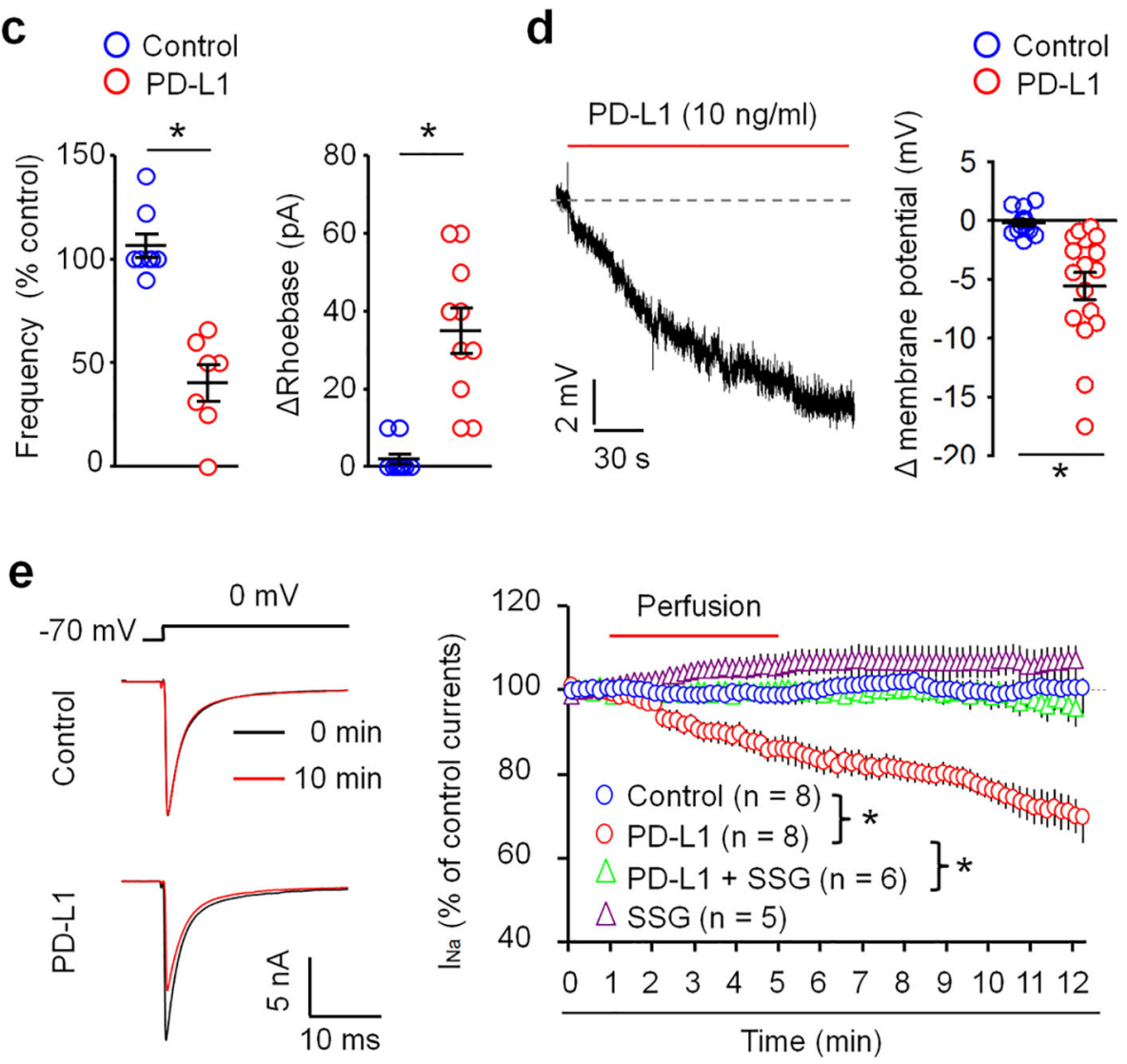

Figure 7. PD-L1 suppresses action potential firing and sodium currents and regulates resting membrane potentials in human DRG neurons

(a) PD-1 immunostaining in a human DRG section. Blue DAPI staining labels all nuclei of cells in DRG. Scale, $50 \mu \mathrm{m}$. (b,c) In vitro patch-clamp recording in dissociated smalldiameter human DRG neurons (30-50 $\mu \mathrm{m})$. (b) Suppression of evoked action potential firing by PD-L1. Insert shows a human DRG neuron with a recording pipette. Scale, $25 \mu \mathrm{m}$. Blue and red arrows show the shift of RMP after the PD-L1 treatment. (c) Percentage change of action potential frequency (left) and rheobase change (right) following PD-L1 perfusion (10 ng/ml). $* P<0.05$, vs. vehicle, Two-tailed Student's t-test, $\mathrm{n}=7-10$ neurons/3 donors. (d) 
Reduction of RMP after PD-L1 perfusion. Right, quantification of RMP change. ${ }^{*} P<0.05$, vs. vehicle, Two-tailed Student's t-test, $\mathrm{n}=13$ and 17 neurons/3 donors. (e) Inhibited of transient sodium currents in dissociated human DRG neurons by PD-L1 $(10 \mathrm{ng} / \mathrm{ml})$ and the effect of SSG $(11 \mu \mathrm{M})$. Left, traces of sodium currents. Right, time course of relative sodium currents showing time-dependent inhibition by PD-L1. $* P<0.05$, Two-Way repeated measures ANOVA, $n=5-8$ neurons/2 donors. Data are mean \pm s.e.m. 
a

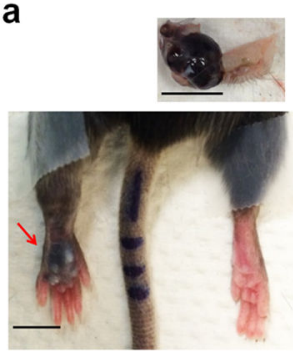

$4 \mathrm{w}$ after tumor inoculation

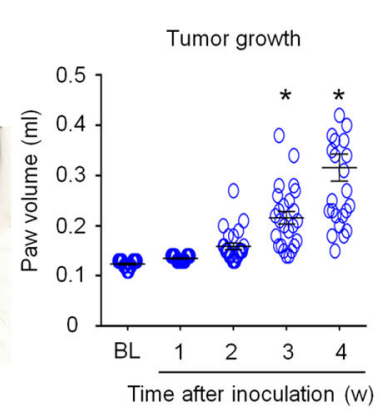

b

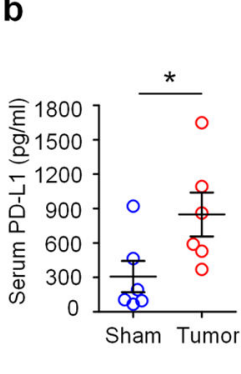

c
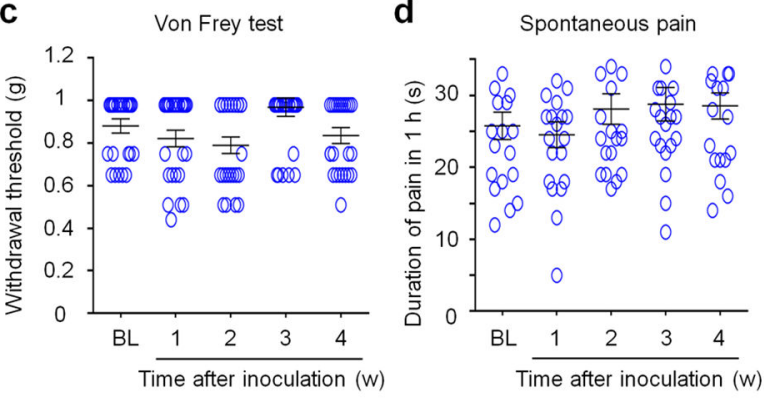

e

f

Ongoing pain

g

Von Frey test

O NT siRNA, $2 \mu \mathrm{g}$

$O$ Vehicle

O sPD-1, $5 \mu \mathrm{g}$
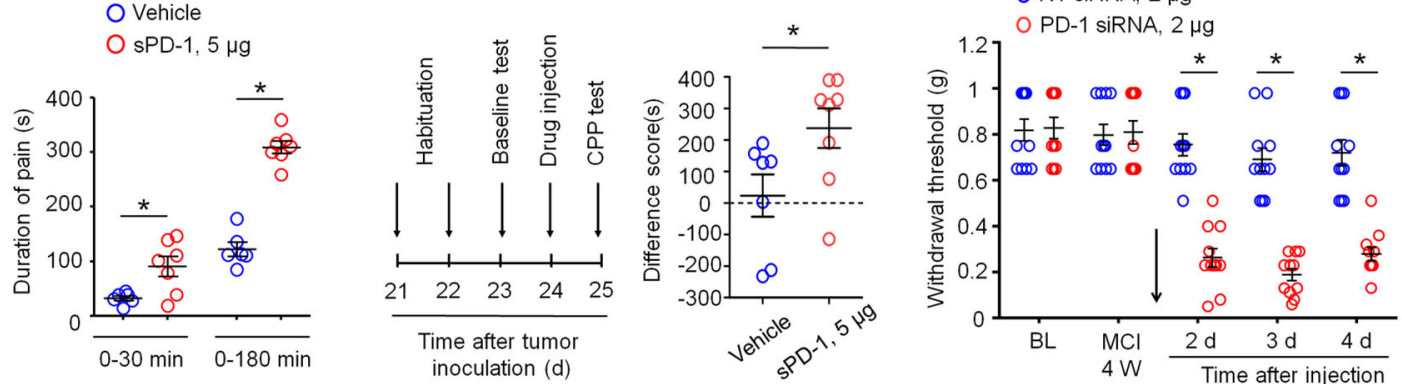
O NT siRNA, $2 \mu \mathrm{g}$ O PD-1 SiRNA, $2 \mu \mathrm{g}$

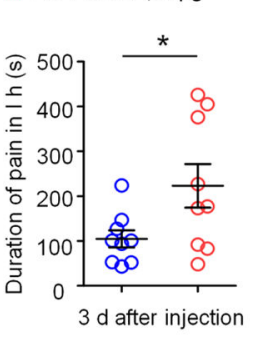

i

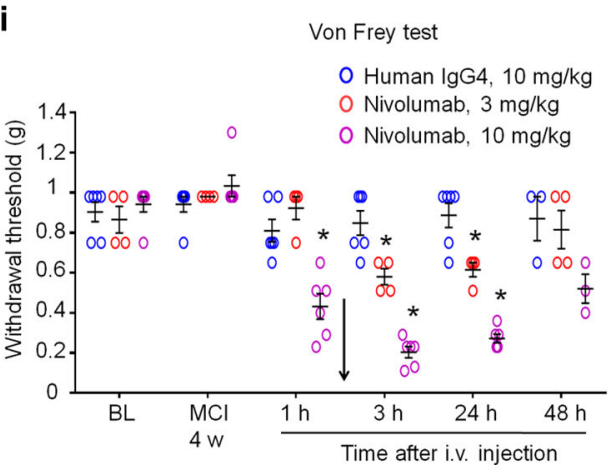

j Spontaneous pain

$\mathbf{k}$

O Human $\lg G 4,10 \mathrm{mg} / \mathrm{kg}$ Human $\lg G 4,10 \mathrm{mg} / \mathrm{kg}$, i.v.

O Nivolumab, $10 \mathrm{mg} / \mathrm{kg}$

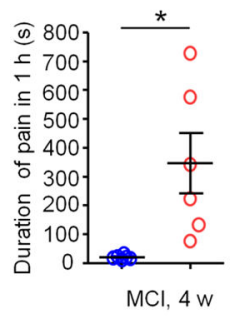

I

Nivolumab, $10 \mathrm{mg} / \mathrm{kg}$, i.v.

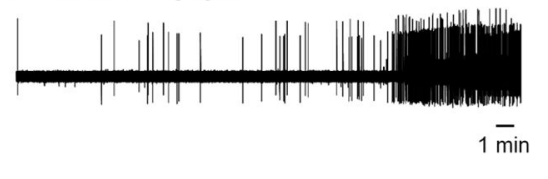

Figure 8. Blocking of PD-L1 or PD-1 signaling induces spontaneous pain and allodynia in a mouse melanoma model

(a) Tumor growth after melanoma cell inoculation (MCI) in a hindpaw. Left, images of ipsilateral hindpaw (red arrow) and contralateral hindpaw and an isolated melanoma (top) at MCI-4w. Scales, $5 \mathrm{~mm}$. Right, time course of tumor growth after MCI, revealed by hindpaw volume change. $\mathrm{BL}$, baseline. ${ }^{*} P<0.05$, vs. $\mathrm{BL}$, One-Way ANOVA, $\mathrm{n}=25$ mice/group. (b) Serum PD-L1 levels in sham control mice and melanoma-bearing mice (MCI-4w). $* P<0.05$, two-tailed Student's t-test. $n=6$ mice/group. (c,d) Time course of mechanical pain (c) and spontaneous pain (duration of licking/flinching, d) after MCI. Note that tumor growth is not associated with the development of mechanical allodynia and spontaneous pain. $n=21$ and 25 mice/group. (e) Induction of spontaneous pain by soluble PD-1 (sPD-1) following i.pl. injection at MCI-4w. Note a rapid onset of spontaneous pain by sPD-1 within $30 \mathrm{~min}$. $* P<0.05$, compared with vehicle, two-tailed Student's t-test. $\mathrm{n}=6$ and 7 mic/group. (f) Induction of ongoing pain (CPP) in melanoma-bearing mice by sPD-1 (i.pl.). Left, paradigm for assessing CPP in a two-chamber test. Right, difference in time spent in drug-paired 
compartment between the pre-conditioning and post-conditioning phases. ${ }^{*} P<0.05$, twotailed Student's t-test, $\mathrm{n}=7-8$ mice/group. (g,h) Induction of mechanical allodynia (g, n=11 mice/group) and spontaneous pain ( $\mathrm{h}, \mathrm{n}=9$ mice/group) by peri sciatic injection of PD-1targeting siRNA $(2 \mu \mathrm{g})$ but not by control non-targeting siRNA (NT, $2 \mu \mathrm{g})$, given at MCI-4w. $* P<0.05$, repeated measures Two-Way ANOVA (g) and two-tailed Student's t-test (h). (i,j) Intravenous Nivolumab ( 3 and $10 \mathrm{mg} / \mathrm{kg}$ ), given at MCI-4w (indicated with an arrow), induces mechanical allodynia (i, $\mathrm{n}=4-6$ mice/group) and spontaneous pain $3 \mathrm{~h}$ after the injection ( $\mathrm{j}, \mathrm{n}=6$ mice/group). $* P<0.05$, compared with control human IgG4, repeated measures Two-Way ANOVA (i) and two-tailed Student's t-test (j). (k,l) Intravenous Nivolumab $(10 \mathrm{mg} / \mathrm{kg}$, MCI-4w) increases spontaneous firing of afferent fibers in the sciatic nerve $3 \mathrm{~h}$ after the injection. (k) Traces of discharges in melanoma-bearing mice treated with Nivolumab and human IgG4 control. (l) Number of spikes in 2 hours after the treatment. $* P<0.05$, two-tailed student's t-test, $\mathrm{n}=5$ mice/group. Data are expressed as mean \pm s.e.m. 